\title{
The Impact of Star Formation on the Interstellar Medium in Dwarf Galaxies: II. The Formation of Galactic Winds
}

\author{
Crystal L. Martin ${ }^{1,2,3,4}$
}

\begin{abstract}
Images and longslit, echelle spectra of the $\mathrm{H} \alpha$ emission from 14 dwarf galaxies and M82 have been used to identify expanding shells of ionized gas. Supershells (radius $>300 \mathrm{pc}$ ) are found in 12 of the dwarfs. The measured shell sizes and expansion speeds constrain the ages and power requirements of the bubbles. The dynamical age of the larger bubbles is typically about $10 \mathrm{Myr}$, and ionized shells older than $20 \mathrm{Myr}$ are rare. An energy equivalent to 100 to 10,000 supernova explosions over this period is needed to drive the shock front that sweeps out the cavity. The current star formation rates are high enough to meet these power requirements. Many of the shells will breakthrough the surrounding layer of HI supersonically, but the projected expansion speeds are typically less than the lower limits on the escape velocity. Some of the shell material may permanently escape from a few galaxies such as NGC 1569. Whether bound to the galaxy or not, these outflows probably play an important role in regulating the star formation rate and are expected to significantly influence the chemical evolution of the galaxies. The shells lift gas out of the disk at rates comparable to, or even greater than, the current galactic star formation rates. They will only displace a substantial fraction of the interstellar gas if their duty cycle is much longer than the rotational period of the disk.
\end{abstract}

\section{Introduction}

The interplay between massive stars and the interstellar medium (ISM) plays a fundamental role in the formation and evolution of galaxies. In addition to ionizing

\footnotetext{
${ }^{1}$ Hubble Fellow

${ }^{2}$ Steward Observatory, University of Arizona, Tucson, AZ 85721

${ }^{3}$ Currently, Space Telescope Science Institute, 3700 San Martin Drive, Baltimore, MD 21218

${ }^{4}$ Visiting astronomer Kitt Peak National Observatory .
} 
radiation and newly synthesized elements, massive stars deliver kinetic energy and momentum to the surrounding gas through stellar winds and supernova explosions. Shock waves driven by an ensemble of massive stars may trigger additional star formation and/or sweep the interstellar gas out of the region actively forming stars (Tenorio-Tagle \& Bodenheimer 1988). The gas flows create a turbulent pressure which helps support the weight of the ISM (e.g. McKee 1990) and cavities which apparently enhance the distance ionizing radiation propagates (Hunter \& Gallagher 1997; Martin 1997).

This feedback from star formation may have a particularly strong influence on the evolution of low mass galaxies. Owing to their low escape velocity, Larson (1974) suggested that the loss of supernova-heated gas would begin earlier and carry away a larger fraction of their initial mass. This idea was further developed by Dekel \& Silk (1988) who used the supernova feedback to regulate the star formation history of the evolving dwarfs. Their starburst-driven wind models were consistent with the observed mass-metallicity and mass-radius scaling relations of dwarfs when a halo similar to those produced in cold dark matter cosmological simulations was included. Mass loss has subsequently been proposed to explain a number of peculiarities about dwarf galaxies such as their abundance patterns (Marconi,Matteucci, \& Tosi 1994) and rapid evolution at moderate redshifts (Phillipps \& Driver 1995; Babul \& Rees 1992). The ejection of the ISM may not be as easy as previously thought, however. In particular, the rupture of a supershell perpendicular to a galactic disk may vent much of the energy leaving most of the disk intact (DeYoung \& Heckman 1994).

Observations of dwarf galaxies reveal an environment conducive to the growth of large bubbles. Their rotation is typically nearly solid body, so shells are not sheared apart; and metallicities are generally sub-solar so cooling times are longer. Indeed, small bubbles permeate the star forming regions of the Magellanic Clouds, and a hierarchy of giant shells $(R<300 \mathrm{pc})$ and supergiant shells $(R \geq 300 \mathrm{pc})$ is plainly visible (Davies, Elliot, \& Meaburn 1976; Meaburn 1980; Kennicutt et al. 1995). The formation of regions like 30 Doradus, which will evolve into a supergiant shell (Chu \& Kennicutt 1994), may be thought of as the first step in the formation of a galactic outflow. Deep imaging of the ionized gas in other dwarfs yields a plethora of candidate structures for supergiant shells. Indeed, roughly one out of every four high-surface brightness dwarfs exhibit at least one shell and/or filaments (Hunter et al. 1993). It is not always obvious, however, which arcs and filaments will show the kinematic signature of an expanding shell (Hunter \& Gallagher 1990).

The kinematic evidence is mounting that some shells do breakthrough the ambient neutral gas. In the LMC, for example, the kinematics of many supershells are surprisingly quiescent compared to the giant shells (Hunter 1994). Some of these supershells are 
believed to be the inner ionized surface of cylindrical HI holes (Meaburn 1979; Meaburn 1980; Hunter 1994), which may have formed as a superbubble blew out perpendicular to the galactic plane. In another Magellanic irregular galaxy, NGC 4449, the very large HI hole may be associated with a shell that expanded out of the galactic plane (Hunter \& Gallagher 1997). In less luminous galaxies like the blue compact dwarf NGC 1705, the expansion of the shell around the central starburst is decidedly non-spherical (Meurer et al. 1992). The kiloparsec scale, expanding shells in amorphous dwarfs (Marlowe et al. 1995) and the faint galaxy IZw18 (Martin 1996) also seem to be elongated in the general direction of the HI minor axis. At issue, however, is whether any of these disk outflows develop into freely flowing winds in which the gas actually escapes from the gravitational potential of the galaxy. Only one member of Marlowe's sample, NGC 3955, was a strong wind candidate. The most convincing arguments for actual mass ejection are based on the detection of X-ray emitting gas well above the galactic plane of NGC 1569 (Heckman et al. 1995). The association of a hot bubble with the cavity formed by the expanding network of extended $\mathrm{H} \alpha$ filaments is reminiscent of the minor axis outflow from M82 (Bland \& Tully 1988; Strickland et al. 1996; Shopbell et al. 1997), although it is not yet clear whether the dynamics of these two classes of galactic outflows are completely analogous.

A more extensive kinematic census is desired to assess the frequency of blowout and the amount of mass loss. This paper presents a catalog of large-scale expanding structures in 14 nearby dwarfs. Although M82 does not strictly meet the sample selection criteria, it was added to the sample to provide a common galaxy between this study and studies of superwinds from more luminous starbursts (Heckman, Armus, \& Miley 1990, hereafter HAM). Galaxies were selected from a volume of radius $d \leq 10 \mathrm{Mpc}$, right ascension $4 h \leq \alpha \leq 14 h$, and declination $\delta \geq-35^{\circ}$. An effort was made to pick the galaxies with the most intense star formation over a range in absolute luminosity from $M_{B}=-13.5$ to $M_{B}=-18.5$. Each radial velocity field was sampled with deep, high-resolution spectra of

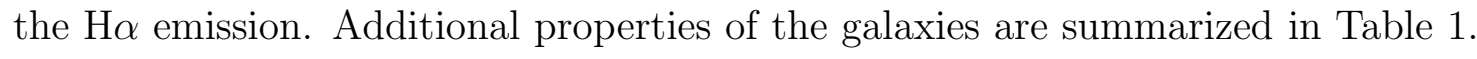

While similar scale shells are found throughout the sample, the net impact on the host galaxy's evolution may be quite varied. Two factors which largely determine the bubble's fate - i.e. the distribution of the HI and the gravitational potential - are not at all uniform across the sample. Hence, the prospects for mass ejection are discussed on a galaxy by galaxy basis. The results have interesting applications for the chemical evolution of dwarf galaxies and the regulation of their star formation rate.

This paper is organized as follows. The observations and data reduction are described in $\S 2$. Section 3 describes the kinematics of the ionized gas, and $\S$ 国 discusses the dynamics of the expanding shells. Rotation curves are sketched in $\S$, and the shell expansion speeds 
are compared to the escape velocity. Section 6 summarizes the main results.

\section{Observations}

Narrowband CCD images of the galaxies at $6580 \AA$ and $6450 \AA$ were obtained at the Steward Observatory 2.3m telescope (Table 2). After standard processing to remove fixed pattern noise (Rieke 1994), the continuum images were subtracted from the corresponding $\lambda 6580$ images to produce images of the $\mathrm{H} \alpha+[\mathrm{N} \mathrm{II}]$ line emission. These images were flux calibrated using observations of standard stars. Photometry of the HII regions agrees with other published values to better than $20 \%$ in all cases, and most of the discrepancy is thought to be caused by slight differences in aperture. The images are sensitive to surface brightnesses $\Sigma(\mathrm{H} \alpha+[N I I]) \geq 4 \times 10^{-17} \operatorname{ergs~s}^{-1} \mathrm{~cm}^{-2} \operatorname{arcsec}^{-2}$ over areas of a few square arcseconds.

The structure of the ionized gas was used to select slit positions for measuring the gas kinematics. The number of observations and positioning strategy varied based on the size of the galaxy and was revised during the course of each observing run based on initial results. In general, most of the large-scale shells and filaments were sampled, and the spatial extent of kinematically interesting features was determined by follow-up observations with the slit rotated $90^{\circ}$. The positions of the slits are identified by number on the images in Figure 2 .

Longslit spectra of the $\mathrm{H} \alpha+[\mathrm{NII}]$ emission lines were obtained using the echelle spectrograph on the KPNO $4 \mathrm{~m}$ telescope with the Tek $2 \mathrm{k} \times 2 \mathrm{k}$ CCD. The instrumental setup, described in Martin \& Kennicutt (1995), provided $11 \mathrm{~km} \mathrm{~s}^{-1}$ resolution (FWHM of the night sky lines) and a usable slit length of $\sim 3.5$. When observing conditions were good, three $1200 \mathrm{sec}$ exposures were sufficient to remove cosmic rays and reveal the line profile of the faintest emission detected in the $\mathrm{H} \alpha$ images. The CCD frames were reduced in the standard way (e.g. Martin \& Kennicutt 1995), and the wavelength calibration is accurate to better than $0.1 \AA$. The narrow emission lines from the night sky have been left in the echellograms in Figure 1 1 to illustrate the accuracy of the distortion correction and the spectral resolution. The spatial resolution varies from $1^{\prime \prime}$ to 2 .'

\section{The Kinematic Atlas and Supershell Catalog}

Figures 1 1 a-l present a kinematic atlas of the $\mathrm{H} \alpha$ echellograms, grouped by galaxy and arranged in a spatial sequence. Even a quick inspection immediately reveals a wealth of structure in the velocity field. Finding and cataloging the large-scale kinematic structures in 
the warm, ionized gas is, however, a several step process. I searched for "Doppler ellipses" on individual echellograms, then located their positions on the $\mathrm{H} \alpha$ images. In almost every case, the kinematically selected regions correspond to shells or filaments in the image. (Note that the converse of that statement does not always hold.) Hence, a two-dimensional picture of the large scale line-of-sight velocity field can be built up by combining the measurements from several slit positions and the morphology of the $\mathrm{H} \alpha$ image. In this section, I draw attention to examples of kinematic structures at individual slit positions, then build a picture of the large-scale kinematic structures in each galaxy.

\subsection{Doppler Ellipses}

In Figure 1, all regions with two or more peaks in the $\mathrm{H} \alpha$ line profile have been marked. Region D near the west end of NGC 4449-9 and feature A along slit NGC 3738-2 and are good examples of the kinematic signature of expanding shells on small angular scales. Their elliptical shape in the echellograms is attributed to the Doppler shift of the emission from the approaching and receding sides of an ionized shell (e.g. Osterbrock 1989 Figure 6.6). I extend the concept of a "Doppler ellipse" to include features like region A along NGC 3077-5 where only partial segments of the ellipse are visible. Polar bubbles like shell A along NGC 3077-5, shell S in I Zw 18-1, or shell B in NGC 4449-3 - are critical to the discussion in this paper. They have two defining characteristics: (1) the associated starburst is located near one end of the Doppler ellipse rather than at its center and (2) the blue-shifted and red-shifted sides of the line profile have different intensities. In the most extreme cases, M82-2 and NGC 1569-10, the magnitude of the line splitting increases with projected height above the plane of the galactic disk, and the two velocity components are not observed to re-converge to a single component.

The expansion velocity and diameter of each shell in Figure 1 were estimated from the maximum amount of line splitting measured along the Doppler ellipse and the length of the region of split lines respectively. When only part of a Doppler ellipse was plainly visible, an ellipse was fit to the two-dimensional line profile of the region marked in Figure 1, and the expansion velocity and shell diameter measured from the lengths of its axes.

\subsection{Reconstructing the Global Gas Kinematics: Example NGC 3077}

Figures 2a-l illustrates the location of the supershells which have been identified and catalogued in Table 3. The solid ellipses show the regions where the Doppler ellipses were 
detected in the echellograms. Their ellipticity is indicative of the dynamical age of a shell. The dotted lines outline the entire complex of warm-ionized gas that I associate with a single expanding bubble.

For example on the $\mathrm{H} \alpha$ image of NGC 3077 in Figure 2a, ellipses are drawn at the location along slit position NGC 3077-5 where shells A and B are detected kinematically. The spatial axis of the ellipse is indicated by a solid line, and the velocity axis is scaled such that 0.3 is $0.1 \AA$ of Doppler shift. A bright loop of emission extends south-eastward from the starburst, but shell A extends out to a fainter loop $46^{\prime \prime}(\sim 800 \mathrm{pc})$ beyond the starburst.

The perimeter of this fainter loop defines complex A in Figure 2. Complex B, just west of the galaxy, is also detected kinematically along positions NGC 3077-2 and NGC 3077-3. Kinematic detections of the same complex along several position angles reduce the need to resort to morphology to constrain the area of the bubble, so the slits with kinematic detections are listed in col. 2 of the supershell catalog (Table 3).

The filaments in complexes $\mathrm{G}$ and $\mathrm{J}$ are clearly the limb brightened edges of the polar bubbles seen on each end of NGC 3077-2. The region of line-splitting labeled shell J extends $34^{\prime \prime}(590 \mathrm{pc})$ south of the starburst and a fainter loop protrudes further to $54^{\prime \prime}(940 \mathrm{pc})$ in the image. These bubbles, like A and B, have apparently pushed their way out of the star forming region in the direction of the least resistance from the ambient gas. In the catalog, the velocity in col. 3 is $1 / 2$ the maximum line splitting measured across the face of the bubble. The projected height of each bubble above the starburst region is given in cols. 4 and 5. Column 6 lists the average $\mathrm{H} \alpha+[\mathrm{NII}]$ surface brightness within the complex.

In contrast, the "radius" listed for shell D, which is coincident with the bright ring of emission just north of the starburst, is $1 / 2$ its diameter. The circular shape of the shell, its higher velocity, and the presence of interior continuum emission are consistent with a younger shell that is still roughly spherical in shape. Such geometrical assumptions introduce some subjective judgement in the cataloged radius at the level of a factor of two. No attempt was made to correct $R$ and $v$ in Table 3 to a true height and expansion velocity based on the inclination of the polar bubbles to our line-of-sight.

The inclination of the polar bubbles can be constrained, however, when the gas dynamics are fully modeled on a galaxy by galaxy basis. Any density gradient in the shell along the polar axis leads to an intensity difference between the velocity components if the polar axis is inclined to our line-of-sight (e.g. HAM 1990; Martin 1996). The shape and density structure of the polar shell determine the relative intensities and velocity offsets of the blue-shifted and red-shifted components of the line. Applied to NGC 3077, the relative 
faintness of the red-shifted component across bubbles $\mathrm{G}$ and $\mathrm{J}$ suggest these lobes are tipped into the plane of the sky. The polar axis of bubble A is likely in the plane of the sky.

\subsection{Detections of Large-Scale, Expanding Structures in Individual Galaxies}

\subsection{1. $N G C 4214$}

Several bubbles are found to be associated with the young star forming region in NGC 4214 (Sargent \& Filippenko 1991; Leitherer et al. 1996). About one-half the ultraviolet light from the starburst comes from the $4-5 \mathrm{Myr}$ old cluster NGC 4214 \#1 which lies within the southern edge of a circular ring in the $\mathrm{H} \alpha$ emission (Leitherer et al. 1996). This ring has a diameter of 8".3 (145 pc) and is identified as complex A in Figure 2. Echellogram NGC 4214-1 shows the $\mathrm{H} \alpha$ emission across it splits into two velocity components near the location of the peak continuum emission. Hence, the ring is probably the projection of the shell surrounding an expanding cavity. The shell must not be spherical, however, since the diameter of the Doppler ellipse is over twice that of the ring in the image. The line-splitting along the edge of shell A at positions NGC 4214-3 and NGC 4214-2 confirm that the area covered by the bubble is larger than shell A itself. The obvious explanation is that our line-of-sight is parallel to the polar axis of a wasp-waisted bipolar bubble, and the shell is brightest where its expansion has been restricted by the higher ambient density in the galactic plane.

Two polar bubbles are detected along NGC 4214-1 and seem to be breaking out of region A. Bubble B is surrounded by some gaseous filaments in Figure 2, although only diffuse emission is seen in the region of complex C. Some faint filaments are found within complex $\mathrm{F}$, although the line-splitting is confined to a few patches with a faint component redshiftd to higher velocity. These wisps reach velocities $\sim 100 \mathrm{~km} \mathrm{~s}^{-1}$ and are unresolved in the spatial dimension; their physical interpretation is less certain than that of the well-defined Doppler ellipses.

By observing the overall tilt of each NGC 4214 echellogram, notice that the rotation of the ionized gas contributes less to the width of the integrated $\mathrm{H} \alpha$ line profile than these shells and wisps. The largest gradient in the central velocity of the line profile is $38 \mathrm{~km} \mathrm{~s}^{-1}$ across NGC 4214-4. The HII complex one arcminute west of the central starburst has a lower velocity than the eastern side of the galaxy. Across NGC 4214-1, NGC 4214-2, and NGC 4214-3, the ionized gas at the northeast end of the slit is moving 15 $-26 \mathrm{~km} \mathrm{~s}^{-1}$ faster than that at the southwest end, so the rotation axis of the ionized gas is closer to the major axis of the galaxy than the minor axis. The HI rotation axis and major 
axis are also oriented at $\mathrm{PA} \approx-20^{\circ}$ (McIntyre 1996).

\subsection{2. $N G C 4861$}

Figure 2 2 c illustrates the bipolar outflow discovered in NGC 4861. Shells A and C detected along NGC 4861-4 coincide with webs of gaseous filaments extending about 1 kpc westward and eastward, respectively, from the giant HII complex in NGC 4861. The reversal in the shape of the line profile between shell $\mathrm{A}$ and shell $\mathrm{C}$ - i.e. the intensity of the blueshifted component is higher across shell $\mathrm{A}$ while the redshifted component is more prominent across shell $\mathrm{C}$ - is consistent with the polar axes of these two bubbles being tipped in opposite directions from our sightline. This outflow axis is not aligned with the minor axis of the continuum isophotes which extend north-eastward in the direction of the smaller HII regions visible in the image.

The kinematics across the two bright loops to the north of the HII complex are less impressive. The line profile across the eastern loop shows a faint red wing, labeled complex B; and shell A seems to partially overlap the line-of-sight through the western loop.

\subsection{3. $M 82$}

The nuclear starburst in M82 drives a bipolar outflow of extraordinary scale along the galaxy's minor axis (Bland \& Tully 1988; HAM 1990). In Figure 2, the filaments extend to a projected height of $240^{\prime \prime}(4.2 \mathrm{kpc})$ above the disk. Residuals from the continuum light of the stellar disk are visible at a $\mathrm{PA} \sim 68^{\circ}$ in the image, and dense gas in this disk apparently confines this superwind in the galactic plane. Echellogram M82-2, along the polar axis of the outflow, is clearly double-peaked on both sides of the nucleus. The maximum separation of these components is $313 \mathrm{~km} \mathrm{~s}^{-1}$ and $270 \mathrm{~km} \mathrm{~s}^{-1}$ respectively, on the northern and southern sides of the slit - in good agreement with the measurements of HAM. These higher resolution data show more variation in the line profile and flux-weighted central velocity with radius than those discussed by HAM. However, the maximum intensity still shifts from the redshifted component (south) to the blueshifted component (north) as expected from tipping the polar axis $\sim 35^{\circ}$ away from our sightline (cf. HAM, Fig. 19).

Since the two components of the line profile do not converge to a common velocity along M82-2, I differentiate this structure from a closed Doppler ellipse in Figure 2. The spatial axis begins where the line profile splits into two components and continues as far as one side of the cavity is detected. A flag is drawn at the last position where both 
components of the double-peaked profile are detected, and its length represents one-half the magnitude of their velocity separation. The filaments south of M82 do appear to converge about 190" south of the starburst in Figure 2, so the bubble may be capped at the end.

The far side of the outflow was observed at a second slit position oriented perpendicular to the polar axis of the wind. Echellogram M82-4 reveals the width of the expanding cavity and Figure 2 shows its close correlation with the morphology of the extended filaments. The faint substructure interior to the big Doppler ellipse appears to form at least three and possibly four smaller ellipses, so the wind is apparently composed of several adjacent cells. These interior walls give the superwind a cellular structure, and I suspect they may be formed by smaller bubbles merging together to form the outflow.

\subsection{4. $N G C 3738$}

Three Doppler ellipses were identified along NGC 3738-2. Of these, shell A has the highest surface brightness and largest velocity, $37 \mathrm{~km} \mathrm{~s}^{-1}$, along our line-of-sight. Complex A was associated with the bright ring of $\mathrm{H} \alpha$ emission in Figure 2 because an even larger region of line-splitting is detected kinematically along NGC 3738-3. The filaments that Hunter \& Gallagher (1990) labeled 1 and 2 in their echellogram are probably the signature of this expanding complex. The radius of bubble A in Table 3 is one-half the diameter of this shell, or $R=8$ ".9 (170 pc). The Doppler ellipse labeled shell B coincides exactly with a faint $\mathrm{H} \alpha$ ring of diameter 6".8 (131 pc). The radius of bubble $\mathrm{C}, 14^{\prime \prime} .9$ (288 pc), is taken as the projected distance from the brightest HII region to the faint arc just beyond the end of the Doppler ellipse.

\subsection{5. $N G C 2363$}

NGC 2363 is a giant HII region on one end of the dwarf galaxy NGC 2366. Roy et al. (1991) have mapped the line profile of its [OIII] $\lambda 5007$ emission using a scanning Fabry-Perot interferometer. Echellogram NGC 2363-4 intersects their expanding shell and associated chimney. Surprisingly this echellogram shows no line-splitting. The mean velocity of the $\mathrm{H} \alpha$ line does, however, increase by $43 \mathrm{~km} \mathrm{~s}^{-1}$ from south to north; and this velocity shear is similar to that measured in [OIII] $\lambda 5007$ by Roy et al. The wings of the line profile broaden to $\sim 500 \mathrm{~km} \mathrm{~s}^{-1}$ FWZI at positions a few arcseconds north and south of the peak $\mathrm{H} \alpha$ intensity. These two sources are only marginally resolved. My spectra are not very sensitive to extremely broad lines (e.g. Roy et al. 1992), since the dispersion is high 
and the spectral coverage is only $\sim 4000 \mathrm{~km} \mathrm{~s}^{-1}$.

Along NGC 2363-5 line-splitting is plainly visible along the base of the Roy et al. chimney - shell B in Figure 11. In Figure 2 $\mathrm{g}$, the bright pair of filamentary loops northnorthwest of the HII region define the boundaries of the associated bubble, complex B. Echellograms NGC 2363-6 and NGC 2363-5 bisect these loops. Although they show line splitting of magnitude $\frac{1}{2} \Delta v \approx 40 \mathrm{~km} \mathrm{~s}^{-1}$ across the eastern half of this complex, the Doppler ellipses extend much further eastward across the fainter arcs labeled bubble A. The limb of shell $\mathrm{A}$ is $34^{\prime \prime}$ (590 pc) from the eastern HII region and also shows a double-peaked profile along NGC 2363-5 where $\frac{1}{2} \Delta v \approx 16 \mathrm{~km} \mathrm{~s}^{-1}$.

\subsection{6. $N G C 2537$}

The Doppler ellipse along NGC 2537-1 coincides with the bright U-shaped HII region on the western side of NGC 2537. Close inspection of the $\mathrm{H} \alpha$ image reveals a faint nebulosity extending 22" (800 pc) westward. A patchy Doppler ellipse is seen over this entire region in NGC 2537-3, so the faint emission comes primarily from an expanding shell.

\subsection{7. $N G C 1800$}

Deep $\mathrm{H} \alpha$ images show a spectacular network of filaments along the minor axis of NGC 1800 (Hunter, Hawley, \& Gallagher 1993; Hunter, van Woerden, \& Gallagher 1994). In Figure 2 $\mathrm{h}$, they extend up to $34^{\prime \prime}(1350 \mathrm{pc})$ north of the nucleus (the peak red continuum emission). Echellogram NGC 1800-2, aligned parallel to the galaxy's major axis, traverses the bright ends of these filaments where they meet the galaxy and reveals a single Doppler ellipse $20^{\prime \prime}$ in diameter. The expansion velocity at the base of the northern polar bubble is $27 \mathrm{~km} \mathrm{~s}^{-1}$. The magnitude of the line splitting reaches $50 \mathrm{~km} \mathrm{~s}^{-1}$ and $43 \mathrm{~km} \mathrm{~s}^{-1}$ in the north and south lobes respectively (Marlowe et al. 1995)

\subsubsection{Sextans A}

As the only member of the Local Group in the sample, Sextans A provides an opportunity to examine the kinematics on a finer scale. Echellogram SexA-1 bisects the central 22" (140 pc) × 35" (222 pc) ring of ionized gas along its long axis (cf. Figure $2 \mathrm{i})$. Three regions of line-splitting are identified. In the northern part of the ring, Doppler ellipse A extends $+69 \mathrm{~km} \mathrm{~s}^{-1}$ toward longer wavelengths and $-48 \mathrm{~km} \mathrm{~s}^{-1}$ toward lower 
velocities. Hunter \& Gallagher (1992) have previously identified the red half of this shell. Doppler ellipse B partly overlaps the blue-shifted component of ellipse A but extends to $-84 \mathrm{~km} \mathrm{~s}^{-1}$, while its redshifted component, visible in a low contrast image stretch, extends to only $+40 \mathrm{~km} \mathrm{~s}^{-1}$. It is not absolutely clear whether these two ellipses are produced by the same expanding structure. They are catalogued as one expanding complex, $v=1 / 2 \triangle v \approx 60 \mathrm{~km} \mathrm{~s}^{-1}$, primarily because of their association inside the the ionized ring in Figure 2 1 . The broad, FWHM $\approx 2 \AA$, wisps of emission in Region $\mathrm{C}$ are separated from the main component of the line profile by +90 and $-110 \mathrm{~km} \mathrm{~s}^{-1}$.

\subsubsection{I $Z$ W 18}

A bubble with radius $r=970 \mathrm{pc}$ and projected expansion velocity $v=34 \mathrm{~km} \mathrm{~s}^{-1}$ was found south-southwest of the northwest HII region. A kiloparsec-scale loop of $\mathrm{H} \alpha$ emission also extends north-northeastward from it, although the kinematic evidence for expansion is less secure. Notice how similar the velocity structure in I Zw 18-1 is to NGC 1569-11, NGC 1569-10, and even to M82-2. The main difference is that the magnitude of the line splitting is about five times larger in M82. See Martin (1996) for a discussion of the power requirements of these shells and the associated production of metals.

\subsubsection{Non-detections}

Expanding shells were found in all but 3 of the 15 galaxies examined spectroscopically. Although it is difficult to quantify the efficiency of the shell-finding strategy, the high incidence of "shell-like" structures in the images with the kinematically detected shells leads me to believe that few of the large shells were missed. In particular, II Zw 40 and VII Zw 403 are unlikely to contain any supershells. The census is, however, incomplete in several ways. First, structures smaller than the angular resolution element were obviously not detected. Second, faint shells along the sightline to high surface brightness HII regions could be lost in the wings of the emission from the HII region. Third, low intensity emission spread over more than $\sim 2000 \mathrm{~km} \mathrm{~s}^{-1}$ may masquerade as continuum and/or be lost in the detector noise. Finally, although the spatial sampling appears to be sufficient to find the largest shells, some medium size shells must be missed due to the incomplete spatial coverage.

The null detection in NGC 5253 is attributed to this latter effect. Marlowe et al. (1995) did find an expanding shell in NGC 5253 which my slit positions simply miss. They measure 
an expansion velocity of $35 \mathrm{~km} \mathrm{~s}^{-1}$ east of the starburst along $\mathrm{PA}=60^{\circ}$; and their Figure 3 clearly shows line splitting out to $R=43^{\prime \prime} .8$ ( $870 \mathrm{pc}$ ) from the central starburst. These parameters and my measurement of the bubble's surface brightness are included in Table 3 . I have also overlaid the slit positions of my three echellograms and those of Marlowe et al. on Figure 2 $2 \mathrm{j}$. (Note that my NGC 5253-3 and the PA $=120^{\circ}$ observation of Marlowe et al. coincide.) While all five of the longslit observations may have missed the region of maximal expansion on the eastern side, the lack of any Doppler ellipse detection on the western side is very surprising given the spatial sampling. The western filaments are apparently quite quiescent. The average $\mathrm{H} \alpha+[\mathrm{NII}]$ surface brightness in the region outlined in Figure 2 is $1.89 \times 10^{-16} \mathrm{ergs} \mathrm{s}^{-1} \mathrm{~cm}^{-2} \operatorname{arcsec}^{-2}$.

\subsection{Blowout in NGC 1569 ?}

The spectacular emission-line filaments emanating to the north and south of NGC 1569 were discovered on early photographic plates (Hodge 1971; Zwicky 1971) and shown to have residual velocities of $-60 \mathrm{~km} \mathrm{~s}^{-1}$ and $+60 \mathrm{~km} \mathrm{~s}^{-1}$ respectively (de Vaucouleurs, de Vaucouleurs, \& Pence 1974). Kiloparsec scale spurs of diffuse X-ray emission are associated with this system of filaments, and approximately one-half the keV X-ray emission comes from a halo 3 '. by 2 '.2 in size (Heckman et al. 1995). The nonthermal radio emission associated with the halo has a high-frequency cutoff attributed to synchrotron radiation losses following the peak starburst activity (Israel \& deBruyn 1988). Heckman et al. (1995) examined the emission-line profiles along two position angles with a resolution of about $57 \mathrm{~km} \mathrm{~s}^{-1}$ at $\mathrm{H} \alpha$, and argued that the outflow was energetic enough to eject most of the interstellar medium. To study the dynamics of this outflow in more detail, I obtained better spatial coverage of the velocity field at high resolution. The echellograms presented here provide new measurements of: (1) the covering angle of the outflow, (2) the changes in the velocity field with height above the disk, (3) the cellular structure in the outflows, and (4) the velocity field near super star cluster A.

In Figure 2 $2 k$, echellograms NGC 1569-10 and NGC 1569-11 cover the outflow in an "X" pattern centered about 22 " N-NE of super star cluster A. The continuum source in the center of NGC 1569-11 is super star cluster A. Just to either side of it, the line profile has a broad blue wing extending $\sim 160 \mathrm{~km} \mathrm{~s}^{-1}$ to lower velocity. A Doppler ellipse is seen on the redward side. A shell associated with super star cluster A has apparently ruptured at least on the approaching side. The outflow along the chimney walls is coincident with the ring of $\mathrm{H} \alpha$ emission inside the central HI hole (Israel \& van Driel 1990). Several X-ray point sources are also found nearby (Heckman et al. 1995). 
Beyond the starburst region, these two echellograms show very similar amounts of line-splitting over arcminute scales. The kinematic signatures of shell B along NGC 1569-11 and shell D along NGC 1569-10 are almost identical as are those of shell A and shell G to the southwest and southeast respectively. The shape of the double-peaked line profile is reversed in the two hemispheres however. To the south the redshifted component is stronger, but it is weaker than the blueshifted component to the north. Tilting the southern and northern lobes toward and away from our sightline, respectively, will produce such a difference if the shell emissivity declines with scale height.

Four slits oriented perpendicular to the polar axis sample the velocity field in the extended lobes. Three Doppler ellipses were independently identified on each of the three slits traversing the southern lobe. Plotted on the image, the Doppler ellipses stack up inside three shells, which can be followed from slit 7 to slit 4 to slit 13 at a projected height of 640 pc. Three Doppler ellipses were also found along the slit traversing the northern outflow, so three superbubbles may have broken through the HI disk. The structure of the outflows is clearly more complex than a web of warm filaments wrapped around a single hot cavity. (In NGC 1569-7, notice also the double shell/chimney structure across the bright arm of shell A.)

The deeper exposure at NGC 1569-13 was obtained to examine the magnitude of the line-splitting as a function of height above the disk. The largest radial velocities are found in complex A where $v$, defined as one-half the maximum separation between the lowest and highest velocity components, increases from $70 \mathrm{~km} \mathrm{~s}^{-1} 140 \mathrm{pc}$ south of the major axis to $99 \mathrm{~km} \mathrm{~s}^{-1}$ at $h=460$ pc. Slit 13 crosses complex A where the two most prominent filaments are converging, but the faint emission between the arms splits into two velocity components separated by $v=140 \mathrm{~km} \mathrm{~s}^{-1}$. While the curvature of a shell could create a gradient in the projected expansion velocity of this magnitude, it is clear that the shell is not strongly decelerated as it pushes through the galactic halo.

The emerging picture has the northern outflow tipped away from our sightline and bubble A on the near side of the inclined disk, but the line profiles along complex G and D complicate this simple interpretation. Across much of complex G (e.g. NGC 1569-4 and NGC 1569-13), the redshifted component of the double-peaked line is consistently weaker than the blueshifted component, and they are comparable across complex F. The projected velocity difference increases from $48 \mathrm{~km} \mathrm{~s}^{-1}$ (NGC 1569-4) to $77 \mathrm{~km} \mathrm{~s}^{-1}$ (NGC 1569-13) across complex $\mathrm{G}$ but is constant at $\sim 64 \mathrm{~km} \mathrm{~s}^{-1}$ across complex F. One interpretation is that the polar axes of bubbles $\mathrm{G}$ and $\mathrm{F}$ are not parallel to bubble A.

Doppler ellipses from complexes A and B were detected by Heckman et al. (1995) along their $\mathrm{PA}=70^{\circ}$ and $\mathrm{PA}=160^{\circ}$ spectra respectively. Some line-splitting is also seen 
where these slits cross bubbles G, F, and E. Our estimated velocities are generally similar. However, the echellograms show a maximum velocity separation of only $2 v=160 \mathrm{~km} \mathrm{~s}^{-1}$ in the region of complex B (along NGC 1569-11) where H95 report $260 \mathrm{~km} \mathrm{~s}^{-1}$.

These polar outflows cover a large fraction of the projected area of the galactic halo. The Doppler ellipses found along the slits oriented perpendicular to the polar axis contain almost all the line-emission in these regions. The angle subtended by these expanding shells can be measured from their location in Figure 2. From the vantage point of cluster A the northern and southern bubbles both subtend about $\sim 140^{\circ}$.

The radii of these shells in Table 3 are the projected distance of the kinematically detected shell from cluster A. The filaments extend beyond this region and converge toward a curved arc $86^{\prime \prime}(920 \mathrm{pc})$ to the south. The south-southeast spur of X-ray emission appears to be contained within this structure (Heckman et al. 1995). Since the velocity separation of the two components in the line profile is still increasing when the fainter side fades below our detection limit, the shell may have partially ruptured near the top of the bubble. In Figure 8 of Hunter, Hawley, \& Gallagher (1993), a fainter arc is visible at $R \approx 116^{\prime \prime}(1.24 \mathrm{kpc})$; and diffuse emission extends beyond it to $R \approx 190^{\prime \prime}(2.0 \mathrm{kpc})$. The relation of these structures as well as the isolated blob of X-ray emission nearby (Heckman et al. 1995) to the expanding bubbles is not yet clear.

\subsection{NGC 4449}

Excluding M82, NGC 4449 has a higher B-band luminosity than any member of the sample, and its HI mass is actually twice that of M82. While it is awkward to call it a dwarf galaxy, I include it here because it provides a particularly dramatic example of the disturbances caused by a burst of star formation in a young disk galaxy. The galaxy shown in Figure 2 $2 \mathrm{~h}$ has a diameter of 5!6 (5.86 kpc) at the $25 \mathrm{~B}$-mag per square arcsecond isophote which is a mere speck in the HI disk which is $\sim 13$ times larger (Bajaja, Huchtmeier, \& Klein 1994). Much of the ongoing star formation is taking place in a central bar-like region running southwest to northeast, although the recent increase in the star formation rate

north of the bar (e.g. Hill et al. 1994) gives the $\mathrm{H} \alpha$ emission a "T" shape. The entire galaxy is forming stars at the modest rate of about $0.07 \mathrm{M}_{\odot} \mathrm{yr}^{-1}$, but the star formation rate in complex $\mathrm{G}$ alone is comparable to that in all of I Zw 18 or NGC 3738.

Although many filaments and partial shells appear in the $\mathrm{H} \alpha$ image in Figure 2, the location of the larger kinematic complexes is not immediately obvious. Echellograms 1 and 2 were aligned parallel to the bar and offset to its NW side. The Doppler ellipses found on the 
southern half of these slits are associated with shell A, and the peaks in the line profile are separated by as much as $160 \mathrm{~km} \mathrm{~s}^{-1}$. The spatial extent of the line splitting increases from about $30^{\prime \prime}$ at a projected "height" above the bar of $10^{\prime \prime}(170 \mathrm{pc})$ to approximately $45^{\prime \prime}$ at a height of $24^{\prime \prime}(420 \mathrm{pc})$. The double-peaked line profile is particularly prominent where these slits intersect a bright filament previously noticed by Hunter \& Gallagher (1990). The spatial axis of NGC 4449-3 follows this filament and shows a Doppler ellipse extending from the bar to a projected height of $\sim 40^{\prime \prime}$. The projected expansion velocity, $35 \mathrm{~km} \mathrm{~s}^{-1}$, is consistent with the velocity difference measured by Hunter \& Gallagher (1990). The intensity of the blueshifted component is stronger which suggests a polar axis tipped away from our line-of-sight. The geometry may be more complicated than a simple polar outflow, however, as NGC 4449-3 also shows multiple high velocity wisps across the bar. The wisps extend up to $+175 \mathrm{~km} \mathrm{~s}^{-1}$ from the intensity-weighted average velocity. Their envelope has an elliptical shape (labeled feature B), but the wisps do not form a clean Doppler ellipse. Their origin is unclear, so they have not been associated with an expanding complex in Figure 21 or Table 3 .

Southeast of the bar along echellogram NGC 4449-7, Doppler ellipse I is visible across the $\mathrm{H} \alpha$ cavity. The measured expansion velocity, $50 \mathrm{~km} \mathrm{~s}^{-1}$, is consistent with the magnitude of the line-splitting Hunter \& Gallagher measured across their filament 2. Farther south, ellipse H extends to $43^{\prime \prime} .9$ between two bright filaments. The kinematic signatures of complex I and $\mathrm{H}$ are clearly those of expanding shells, and shell-like features are seen in the image. The HI column is also very low within complex I (Hunter \& Gallagher 1997). To the north of these bubbles, patchy line-splitting and wisps are detected up to Doppler ellipse J. It is no clear whether this ellipse is related to complex I or even complex C.

The kinematic activity is not limited to the region near the bar. Echellograms NGC 4449-9 and NGC 4449-11 show Doppler ellipses at the NW end of the "T", hereafter complex D. A larger region of line-splitting labeled "C" in Figure 2 shows several adjacent Doppler ellipses over $D \approx 25$ " region. At the top of the "T", complex G extends from two HII complexes in the west to a faint arc on its eastern boundary. Along NGC 4449-9, Doppler ellipse $\mathrm{G}$ is prominent in the [NII] $\lambda 6458$ line, but the $\mathrm{H} \alpha$ emission is difficult to separate from the bright HII region emission. Along the eastern perimeter of the galaxy, the three Doppler ellipses identified along NGC 4449-10 coincide with partial rings of emission in the $\mathrm{H} \alpha$ image.

All the Doppler ellipses identified on the echellograms are plotted on the $\mathrm{H} \alpha$ image in Figure 2. The associated kinematic complexes are outlined by dotted lines, and several deserve further comment since their boundaries are appreciably less well-defined than the 
other cataloged structures. Features B and J show very disturbed gas motion but not clean Doppler ellipses, so they were not catalogued as expanding shells. Only the faint, fairly diffuse, emission from complex $G$ and complex D shows the kinematic signature of expansion, so the brighter regions were masked out of the apertures for shell photometry. The spatial extent of complexes A, C, and $\mathrm{H}$ were estimated conservatively. Complex A, restricted to the filaments comprising the Doppler ellipses, corresponds very closely to a hole in the HI emission discovered by Hunter \& Gallagher (1997). Arc-shaped filaments extend further, about $1.2 \mathrm{kpc}$, to the northwest but appear relatively quiescent (Hunter \& Gallagher 1997). As suggested by Hunter \& Gallagher, these are probably the inner ionized edge of HI cavities. The X-ray emission in both the soft $(0.12-0.28 \mathrm{keV})$ and hard (0.76-2.02 keV) ROSAT bands does extended in the direction of bubble A well beyond the conservative radius of the Doppler ellipse, 34".5 (600 pc) (Della Ceca et al. 1997). Several bubbles may be merging into a superbubble in the region of complex C. The X-ray map of Della Ceca et al. (1997) also shows two local maxima within complex C. The expansion associated with Doppler ellipse $\mathrm{H}$ probably extends further to the northwest to include the loop of emission identified by Hunter \& Gallagher (1990, i.e. Loop 1). Their Figure 2 panel b shows hints of a Doppler ellipse in the faint component of the line. A prominent finger of very soft X-ray emission appears to curve southward from this complex (Della-Ceca et al. 1997). Overall the picture is one of large shells breaking out of the bar-like region, and many smaller shells growing along the periphery of the disk.

\subsection{Summary: A Catalog of Superbubbles}

Figure 3 illustrates the distribution of bubbles in the radius - velocity plane. Inspection of Figure 3 or Table 3 draws attention to the relatively large number of shells with $R \geq 400 \mathrm{pc}$. The bubbles in seven amorphous dwarf galaxies discussed by Marlowe et al. (1995) have very similar properties. This distribution of cataloged shells is in fact bimodal with maxima at $R<200 \mathrm{pc}$ and $R \approx 850 \mathrm{pc}$. Although the distribution of shell sizes in the Magellanic Clouds has a similar minimum near 750 pc (Meaburn 1980), I find no evidence for bimodality in the morphologically-identified shell sample of Hunter, Hawley, \& Gallagher (1993). One expects my distribution of detected shells to be strongly biased towards the larger complexes since my sampling technique is fairly complete on large scales but may miss many smaller bubbles. The apparent bimodality may therefore be misleading, although it might be used to motivate a kinematic survey with two-dimensional spatial coverage. 


\section{Superbubble Dynamics}

This section compares the power requirements and ages of all the large-scale expanding structures in the catalog. The estimates for individual bubbles are only accurate to factors of a few since the dynamical models ignore the complicated geometry. Two model-independent measurements provide important checks on the validity of the results. The integrated $\mathrm{H} \alpha$ luminosity of each galaxy directly measures the massive star formation rate, and areal $\mathrm{H} \alpha$ photometry of the extended shells indirectly constrains their mass. For individual galaxies, hydrodynamical simulations tuned to the real, non-spherical distribution of gas and mass should yield results accurate to be better than a factor of two in the future.

\subsection{Mechanical Power from Massive Stars}

The interated $\mathrm{H} \alpha+[\mathrm{NII}]$ flux measured from narrowband images obtained at the Steward Observatory Bok Telescope is given in Table 4 . The [N II] $\lambda \lambda 6548,6584$ $/ \mathrm{H} \alpha$ ratio and Balmer decrement were measured from longslit spectra (Martin 1997), and luminosity-weighted corrections were applied to obtain the integrated $\mathrm{H} \alpha$ luminosity. Column 5 shows the corresponding Case B recombination rate at an electron temperature

of $10^{4} \mathrm{~K}$. Assuming no ionizing radiation escapes the galaxy, the recombination rate is equal to the ionization rate - a measure of the number of massive stars. For a stellar metallicity of $0.25 \mathrm{Z}_{\odot}$ and stellar initial mass function with slope $\alpha=-2.35$ from $1 \mathrm{M}_{\odot}$ to $100 \mathrm{M}_{\odot}$, a star formation rate of $1 \mathrm{M}_{\odot} \mathrm{yr}^{-1}$ produces $3.16 \times 10^{53}$ hydrogen ionizing photons per second (Leitherer \& Heckman 1995; LH95). Column 6 of Table 1 lists the star formation rates derived from the global recombination rate. In the LH95 starburst model, the mechanical power reaches an equilibrium value $L_{w}=1.45 L_{\mathrm{H} \alpha}$ after $40 \mathrm{Myr}\left(T_{e}=10^{4} \mathrm{~K}\right)$.

\subsection{Shell Mass}

To photometrically measure the mass in a shell, one needs to know the shell volume and the filling factor of warm ionized clouds. The volume is well constrained by the projected area of the kinematically identified filaments, but the volume filling factor $\epsilon$ is not immediately known to even order of magnitude accuracy. In this paper, $\epsilon$ is treated as a free parameter, but constraints from additional observations will be described in detail in a forthcoming paper.

The line flux from each expanding complex was measured within the aperture shown in Figure 2. These photometric apertures encompass mainly the extended filaments, 
so contamination from foreground/background HII regions was not generally a concern. NGC 4449 was an exception, and HII regions defined by a surface brightness threshold were masked out of the apertures for NGC 4449. The fluxes were corrected for foreground Galactic extinction, $\mathrm{A}(6570)=0.08 \mathrm{mag}(\csc \mathrm{b}-1)$, and [NII] emission. The root mean

square electron density for the complex is then $n_{r m s}^{2} \equiv F_{H \alpha} /\left(h \nu \xi \alpha_{H \alpha}^{e f f}(T)\right)\left(4 \pi d^{2} / V_{P}\right)$, where the number of $\mathrm{H}$ nuclei per free electron, $\xi$, is $\sim 10 / 11$ since most of the He is singly ionized (Martin \& Kennicutt 1997), and the recombination coefficient is evaluated at $T_{e}=10^{4} \mathrm{~K}$ (Osterbrock 1989). The mass of warm hydrogen and helium in the shell, $M=\mu_{e} m_{H} n_{r m s} V_{E} \epsilon^{1 / 2}$, is sensitive to the fraction of the bubble volume filled by warm, ionized filaments. The mass estimates in Table 3 parameterize the filling factor in terms of $\epsilon=0.1$, a value near the high end of the range measured in individual HII regions (e.g. Kennicutt 1984), and are intended to be upper limits.

When $\epsilon$ is better constrained, the bubble volume will be the major source of error in the mass estimate. Two volume estimates are used here to provide some insight into this systematic uncertainty in the shell mass. A volume, $V_{P}$, was derived from the area of the photometric aperture times two-thirds the aperture width. For some shells such as NGC 3077-D, this photometric aperture includes the entire complex, but for polar shells such as NGC 3077-J the base of the shell emerging from the starburst region is clearly not included in the volume estimate. The photometric aperture also overestimates the volume in some cases, since it must extend somewhat beyond the shell to capture all the flux e.g. NGC 4449-E1. To estimate the uncertainty in the mass, shell masses were calculated for both the volume $V_{P}$ and an ellipsoidal model for the bubble volume, $V_{E}$. The length of the major axis of the prolate ellipsoid was set equal to the shell diameter - often the height of the polar axis; and the minor axis length set equal to the shell width. The mass differenence is expressed as a fraction of $M\left(V_{E}\right)$ in parentheses in Table 5 .

In column 7 of Table 3, the kinetic energy of each complex is the product of a complex's ionized mass and half the square of its projected expansion velocity. Ignoring any additional kinetic energy in large, neutral shells (e.g. Meaburn 1980), the kinetic energies in Table 3 are expected to be accurate to a factor of a few. They provide an empirical measure of the kinetic energy in the large-scale expanding complexes that is independent of the details of a dynamical interpretation.

\subsection{Timescales and Power Requirements}

The dynamics of a wind-driven shell provide some basic insight into the ages and power requirements of the supershells. In the simplest model, ejecta from a concentration 
of stellar winds and supernovae push the surrounding interstellar gas away and generate a shock front. This shocked ISM radiates and collapses into a thin shell. A second shock propagates inward through the lower density wind of ejecta creating a very hot, low density bubble. This bubble's pressure drives the shell outward. Neglecting radiative losses from this cavity, the solution for the shell's equation of motion is (Weaver, McCray, \& Castor 1977; Shull 1993):

$$
R=0.762(L / \rho)^{1 / 5} t^{3 / 5}
$$

or

$$
V=0.458(L / \rho)^{1 / 5} t^{-2 / 5}
$$

For a measured shell radius and velocity, these equations define an age $\tau$ and mechanical power $L_{w} / n_{0}$, where $n_{0}$ specifies the ambient hydrogen density $n_{0} \equiv \rho /\left(1.4 m_{H}\right)$. In Figure 4 , dashed, diagonal lines illustrate isochrones for $\tau(R, V)=1,5,10,15$, and $20 \mathrm{Myr}$. For a specified ambient density, the energy injection rate is read off the orthogonal axis. In other words, a shell in a uniform medium evolves along a dotted curve from upper left to lower right when the power supply is steady. The inferred ages and power of each kinematically-detected complex are tabulated in Table 3 columns 9 and 10.

Several conclusions can be drawn from the locus of supershells in Figure 4. Many of the larger shells have ages $\sim 10$ Myr. Since the shells are predominately photoionized by massive stars with lifetimes of only a few million years (Martin 1997), the formation of massive stars has continued for at least several generations in the starburst region. However, no ionized shells with ages greater than $\sim 20 \mathrm{Myr}$ were identified. The energy injection rates range from $L_{i n} / n_{0}=10^{38} \mathrm{~cm}^{3} \mathrm{ergs} \mathrm{s}^{-1}$ to $\gtrsim 10^{42} \mathrm{~cm}^{3} \mathrm{ergs} \mathrm{s}^{-1}$. Winds from normal O stars supply $L_{i n} \sim$ few $\times 10^{36} \mathrm{ergs} \mathrm{s}^{-1}$ (e.g. Chiosi \& Maeder 1986), and the contribution from an average supernova (i.e. $10^{51} \mathrm{ergs} / 10 \mathrm{Myr}$ ) is similar. Hence, many thousands of stars contribute to the formation of the larger bubbles. A typical supershell (800 pc, $50 \mathrm{~km} \mathrm{~s}^{-1}$ ) requires the combined energy of $\sim 10^{3}$ supernovae. The feedback is apparently hierarchical on global scales!

The polar, rather than spherical, geometry of many of the larger shells affects the accuracy of these power and age estimates. No corrections for inclination have been applied, so the measured radius and velocity are systematically lower than the height and maximum expansion velocity of a polar shell. Over such large scales, gradients are also expected in the ambient density. Considering these deviations from the basic model, the parameters derived from the idealized, wind-blown bubble model are probably instructive at the level of a factor of a few.

Figure 5 illustrates one method I have used to monitor the growth of systematic 
errors. In the wind-driven bubble model, the kinetic energy of a swept-up shell is $K E=1.44 \times 10^{52} n_{0} R_{100}^{3} v_{100}^{2}$ ergs, where $R_{100}$ and $v_{100}$ are the shell radius and velocity in units of $100 \mathrm{pc}$ and $100 \mathrm{~km} \mathrm{~s}^{-1}$ respectively. In Figure 5, this estimate is compared to the mass of ionized gas estimated photometrically from the $\mathrm{H} \alpha$ luminosity of the complex. The requirement of consistency constrains the two parameters $n_{0}$ and $\epsilon$. For the assumed values

of $n_{0}=0.1 \mathrm{~cm}^{-3}$ and $\epsilon=0.1$, the agreement is good for the medium-size superbubbles with $r \sim 500$ pc. The kinetic energy predicted by the dynamical model is systematically higher, however, as the shell size increases. One explanation for this divergence is that the average density seen by the expanding shells becomes smaller as the shells get larger. An average density of $0.01 \mathrm{~cm}^{-3}$ would bring the values for the largest shells into agreement.

For an average ambient density of $0.1 \mathrm{~cm}^{-3}$, the dynamical model implies energy injection rates of $L_{W}=\left(10^{-1}(L / n) \mathrm{ergs} \mathrm{s}^{-1}\right.$ as shown on the y-axis of Figure 6. The mechanical power available from supernovae and stellar winds, solid line, was estimated from the ionizing luminosity of the host galaxy. For illustrative purposes, the same conversion from ionizing luminosity to mechanical power was applied to all the galaxies (see $\S 4.1$ ). Since all the complexes lie below the line, the current massive star populations in these galaxies could power even the largest expanding complexes. Unless the star formation rate has increased dramatically during the lifespan of a bubble, the mechanical power liberated by the massive stars is more than adequate to create the observed bubbles.

\subsection{Fate of the Supershells}

Once the massive stars die off, a supershell may in principle continue to coast outward conserving its momentum. Eventually, however, the bubble's environment dictates its fate. I will consider in turn the role of radiative losses, the pressure of the ambient medium, and the gas scale height of the ambient medium. Since dwarf galaxies typically exhibit solid-body rotation, shear forces are expected to be negligible in their disks.

\subsubsection{Cooling Time}

As radiative losses from the bubble's interior become significant, the shell's growth rate will slow down to that of the momentum-conserving solution. In the self-similar solution for the shell's motion, the thermal energy in the bubble is 5/11 of the kinetic energy supplied by the stars, so in terms of a cooling coefficient $\Lambda(T)$ the timescale for the bubble to radiate all its thermal energy is $t_{c} \approx\left(5 / 11 L_{w} t\right) /\left(4 / 3 \pi R_{c}^{3} \Lambda n_{H} n_{e}\right)$. The temperature dependence of 
the cooling rate is fit reasonably well by a power law, $\Lambda=\Lambda_{0} T^{-0.5}$, between temperatures of $10^{5} \mathrm{~K}$ and $10^{7.5} \mathrm{~K}$. At cosmic abundance $\Lambda_{0}$ is $\approx 1.6 \times 10^{-19} \mathrm{ergs} \mathrm{s}^{-1} \mathrm{~cm}^{3}$ (Koo \& McKee 1992), which is probably a good upper limit for $\Lambda_{0}$ in the hot ISM of the dwarf galaxies.

The cooling rate is sensitive to the amount of mass residing in the cavity. The conventional analytic solution for the temperature and density structure inside a bubble is based on classical conductivity theory. Taking the predictions for the bubble's maximum density and temperature as representative of the bubble interior (e.g. Shull 1993), the estimated cooling time is $t_{c} \approx\left(2.46 \times 10^{8} \mathrm{yr}\right) v_{100}^{1 / 7} R_{100}^{5 / 7} n_{0}^{-2 / 7}$. Radiative losses from the bubble interior are a concern when the cooling time is less than or of the same order as the age of the bubble, i.e. when $v \lesssim\left(0.51 \mathrm{~km} \mathrm{~s}^{-1}\right) R_{100}^{1 / 4} n_{0}^{1 / 4} \kappa_{0}^{5 / 8}$. For reasonable values of the ambient density, almost all the shells in Figure 3 lie above this relation. Based on conventional arguments then, radiative losses from the bubble interiors would not have significantly affected the dynamical evolution of the shells. However, "mass loading" by evaporation off entrained clouds may drive the actual mass of hot gas as much as an order of magnitude higher (Martin \& Kennicutt 1995; Heckman et al. 1995; Strickland et al. 1996); and the cooling times may need downward revisions when better X-ray data becomes available.

\subsubsection{Pressure Confinement}

The external pressure of the ISM may also suppress a shell's growth. A bubble is said to be pressure confined when its internal pressure falls to that of the ambient ISM (e.g. Koo \& McKee 1992). This condition is met when the shock speed slows to roughly $13-$

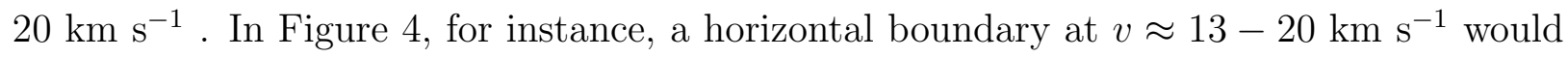
denote the region where pressure confinement becomes relevant to a shell's evolution. All the shells described in $\S 3$ are expanding supersonically, however; so it is not clear that even the larger shells will stall.

\subsubsection{Breakthrough and Blowout}

As a shell outgrows its galaxy, the flattening of the ambient gaseous disk is expected to collimate the outflow along the minor axis (e.g. DeYoung \& Heckman 1995). A shell will breakthrough the disk if it is expanding supersonically when it reaches one gas scale height. Puche et al. (1992) measured an HI scale-height of 625 pc in Ho II , so the gas scale height in dwarf irregulars may typically be larger than that of late-type spirals. For a midplane gas density $n_{0}$, effective scale height $H=500 \mathrm{pc}$, and sound speed $c_{0}=20 \mathrm{~km} \mathrm{~s}^{-1}$, the critical 
energy injection rate for breakthrough is $L_{\text {crit }} / n_{0} \geq 8.0 \times 10^{38} H_{500}^{2} c_{20}^{3} \operatorname{ergs~s}^{-1} \mathrm{~cm}^{3}$. In Figure 4 , the thick dashed line illustrates this critical power for breaking through the disk. Many of the supershells lie above this relation, so the shells are expected to breakthrough the HI disk.

Maps of the HI 21-cm emission support this argument. The HI distribution in three dimensions is often not uniquely determined for dwarf galaxies, but the position angles of the polar shells in Figure 2 are preferentially oriented closer to the minor axis of the HI emission than the major axis. The lack of perfect alignment indicates the collimation occurs on smaller spatial scales. The dimensions of the HI emission, typically at the $10^{19}$ or $10^{20} \mathrm{~cm}^{-2}$ column density contour, are given in Table 1. In NGC 1569, NGC 1800, NGC 2366, IZw18, M82, NGC 3077, NGC 4861, and NGC 5253 the polar bubbles have grown to heights beyond much of the HI disk. The same may be true in NGC 3738, NGC 4214, and NGC 4449, although the low inclination precludes direct observation. Although the prevalence of very low-density HI halos is unknown, Sex A is the only galaxy in the sample with a reported detection. Hence, it would appear that these polar shells will meet little more resistance from the ambient HI disk/halo.

An important question is whether the shells rupture opening a conduit for the escape of the hot gas in their interior. Numerical simulations suggest the shells do not accelerate and fragment from Rayleigh-Taylor instabilities until they reach several scale heights (Mac Low, McCray, \& Norman 1989). The minimum energy injection rates for blowout are therefore "several" times higher than for breakthrough alone. Blowout still seems likely, however, for many of the bubbles in Figure 4 lying above $L / n=10^{40} \operatorname{ergs~s}^{-1} \mathrm{~cm}^{3}$. The fate of this material then depends on the escape velocity several hundred parsecs above the HI disk.

\section{Mass Loss}

Figure 2 shows numerous examples of warm ionized gas being lifted out of the galactic disk. To determine whether this material will escape from the galactic gravitational potential, a model of the distribution of the visible and dark matter is used to calculate the escape velocity. Low-resolution HI maps and rotation curves have been published for most of the galaxies in this study. Beam-smearing artificially flattens the rise of these rotation curves, so it would be misleading to describe fits to the rotation curves. The data do, however, constrain the maximum circular velocity and provide a measure of the Keplerian mass within that radius $r\left(v_{\text {circ }}\right)$ (Table 1). In this section I examine this constraint on a galaxy by galaxy basis and discuss the normalization of the dark (hereafter halo) component. Assuming the halo density profiles resemble the profiles derived empirically 
from low surface brightness galaxies (Burkert 1992), the escape velocity from dark halos extending to roughly the virial radius is easily computed.

\subsection{Galactic Mass Loss? Case Study NGC 1569}

HI rotation curves for NGC 1569 from Reakes (1980) and Israel \& van Driel (1990) are reproduced in Figure 7 . The circular velocity at $r=1 \mathrm{kpc}$ is $\lesssim 31 \mathrm{~km} \mathrm{~s}^{-1}$. The projected expansion speeds of the kiloparsec-scale shells are 1.4 to 4.0 times higher, so NGC 1569 has the largest ratio of shell speed to maximum circular velocity in the sample. It is perhaps the most likely of any of the nearby starbursts to lose a substantial fraction of its warm ionized gas mass to the intergalactic medium, so the limits on its escape velocity are particularly interesting.

The dashed and dotted lines in Figure 7 show the rotation curves for dark halos with core radii of $r_{0}=0.53 \mathrm{kpc}$ and $1.4 \mathrm{kpc}$, respectively. This one-parameter family of models is a good description of the dark matter distribution in the seven low surface brightness galaxies where it has been directly measured (Burkert 1995). The circular velocities of these Burkert halos are significantly lower than observed if one accepts either the turnover in the Reakes rotation curve at $r \sim 1 \mathrm{kpc}$ or the higher velocities in the Israel \& van Driel (1990) data.

The distribution of the extra mass in the inner galaxy significantly affects the extrapolated mass distribution at large radii. The resolution of the HI rotation curve is not sufficient to distinguish between a denser halo model and a disk-like matter distribution, but a maximum disk model can be compared to the visible mass in the disk. For example, the circular velocity in a disk with an exponential surface density profile and a scalelength similar to the B-band surface brightness profile, $\alpha^{-1} \approx 530 \mathrm{pc}$, would reach a velocity of $\sim 34 \mathrm{~km} \mathrm{~s}^{-1}$ at $1.1 \mathrm{kpc}$ for a central surface density of $240 \mathrm{M}_{\odot} / \mathrm{pc}^{2}$. (The central density can be reduced if the rotation curve continues to rise and the disk scalelength is increased.) The B-band surface brightness within one effective radius is $39 \mathrm{~L}_{\odot} \mathrm{pc}^{-2}$ (de Vaucouleurs et al. 1991). For a purely exponential surface brightness profile, the central surface brightness would be about 5 times higher or $\mu_{0} \approx 200 \mathrm{~L}_{\odot} \mathrm{pc}^{-2}$. Although the stellar mass to blue light ratio of the $\sim 10 \mathrm{Myr}$ old burst is very small $(\sim 0.1)$, even a small ( $\gtrsim 10 \%)$ contribution to the light from an old (i.e. several Gyrs) population would raise the integrated $M / L_{B}$ ratio to $\gtrsim 1.1$ (Bruzual \& Charlot 1993). In addition, for $\left(M / L_{B}\right)_{*} \sim 1 \mathrm{M}_{\odot} / \mathrm{L}_{\odot}$, the atomic gas mass, $1.4 M_{H I}$, or $\sim 1.2 \times 10^{8} \mathrm{M}_{\odot}$, exceeds the stellar mass, $L_{B}=1.62 \times 10^{8} \mathrm{~L}_{\odot}$. Some of this material lies beyond a radius of $1 \mathrm{kpc}$, but clearly the Keplerian mass interior to $1 \mathrm{kpc}$ $\left(\sim 2.2 \times 10^{8} \mathrm{M}_{\odot}\right)$ can be accounted for without invoking a dark matter component. 
NGC 1569 would be very unusual, however, if it did not have a dark matter halo; and the dominant contribution from luminous, disk mass at radii less than $1 \mathrm{kpc}$ in no way rules out the presence of a large, diffuse halo. Adding an $r_{0}=1.4 \mathrm{kpc}$ Burkert halo to a disk model with scalelength $500 \mathrm{pc}$, the central density of the latter is only reduced to $155 \mathrm{M}_{\odot}$ $\mathrm{pc}^{-2}$. Even a halo like that around DDO 154, core radius $\sim 2.9 \mathrm{kpc}$, would contribute only $7.8 \times 10^{7} \mathrm{M}_{\odot}$ within $1 \mathrm{kpc}$, although it would cause the rotation curve to turnover at a much larger radius than observed. A substantial range of composite disk plus halo mass distributions are then consistent with the data. The escape velocity from one of them is $v_{\text {esc }}=\sqrt{2} \sqrt{|\phi(R, z)|}$, where the galactocentric radius $R$ is equal to zero above the burst region and $\phi=\phi($ disk $)+\phi($ halo $)$. The halo potential is the integral of the gravitational force on a test mass from radius $z$ to the halo truncation radius. The potential at a height $z$ above the center of an infinitely thin disk can be computed using Toomre's method to solve the Laplace equation (Toomre 1963; Binney \& Tremaine 1987 §2.6).

Figure 8 compares the escape velocity above the galactic center for three models of the mass distribution. The exponential disk model $\left(\alpha^{-1}=500 \mathrm{pc}, \sigma_{0}=240 \mathrm{M}_{\odot} \mathrm{pc}^{-2}\right)$ with no halo is a lower limit on the escape velocity. A more realistic model, dotted line, includes a spherically symmetric Burkert halo with core radius $r_{0}=1.4 \mathrm{kpc}$ and truncation radius $160 \mathrm{kpc}$. Lowering the central disk density to $155 \mathrm{M}_{\odot} \mathrm{pc}^{-2}$ to maintain a circular velocity of $\sim 30 \mathrm{~km} \mathrm{~s}^{-1}$ at $r=1 \mathrm{kpc}$, the escape velocity from the composite potential is raised to almost $100 \mathrm{~km} \mathrm{~s}^{-1}$. The third model has equal mass contributions from the halo and disk components inside $1 \mathrm{kpc}$. Since the halo central density must be increased by a factor of 1.5 over Burkert's $\rho_{0}\left(r_{0}\right)$ relation, this model can be thought of an upper limit on $v_{\text {esc }}$.

The expansion speeds of the polar shells in NGC 1569 are shown as filled circles in Figure 8. The shell velocities range from $40 \mathrm{~km} \mathrm{~s}^{-1}$ to $120 \mathrm{~km} \mathrm{~s}^{-1}$. Several are expanding faster than the escape velocities from the disk model at $z=1 \mathrm{kpc}$. When a halo component is included, however, the expansion of all but one of the shells is sub-critical. Since the projected expansion speeds are $\lesssim v_{e s c}$, these shells are marginally bound. In other words, some shell mass could permanently escape - i.e. corrections for projection effects could easily increase $v$ by $50 \%$, but much of the warm ionized gas probably remains bound to NGC 1569.

\subsection{Escape Velocity Estimates}

As illustrated in Figure 9, the resolution of the HI rotation curves for the other galaxies varies from good, i.e. Sextans A, to essentially a single element, i.e. NGC 4861. Inclination corrections, see Table 1, have been applied, but the low resolution and non-circular gas 
motions may introduce significant errors in the Keplerian masses derived for a few objects. The descriptions below provide a qualitative impression of the possible magnitude of such systematic errors. Array data have not been published for NGC 2537 and NGC 3738, and the three galaxies without superbubble detections are not shown.

The Keplerian mass provides some guidance for the potential models. The difference between the dynamical mass and the mass of atomic gas in Table 5, for example, can only be explained with a stellar component if its mass to light ratio is as high as that in Column 8. The ratios for NGC 1569, NGC 3077, NGC 4214, and NGC 5253 are fairly typical for stellar populations, so a baryonic disk could dominate the potential in the inner few kiloparsecs. In the other dwarfs, however, some form of dark matter apparently dominates the mass in even the regions interior to $R\left(v_{\text {circ }}\right)$. The spatial distribution of the matter is unknown, and a disk of cold molecular gas could contribute much of the mass. To place a conservative upper limit on the escape velocity, however, the extra mass is best modeled as an increase in the central density of the dark halo.

\subsubsection{Sextans A}

Sextans A is one of only three sample members with $M_{B}>-14$ mag and the only member of the Local Group. The rotation curve along the kinematic major axis (Skillman et al. 1988) is shown by the squares in Figure 9. The small ionized shell has an expansion velocity, $\sim 60 \mathrm{~km} \mathrm{~s}^{-1}$, significantly higher than the rotational speed of the neutral gas at the galactocentric radius of the first-ranked HII region. The elliptical orbits of the HI in the inner galaxy have been interpreted as evidence for a gaseous bar (Skillman et al. 1988), and the first-ranked and second-ranked HII regions are located near local maxima in the HI column at opposite ends of this bar. Dotted lines illustrate the rotation curve and escape velocity of an $r_{0}=532 \mathrm{pc}$ Burkert halo. An increase of a factor of 5.3 in the halo central density produces a better fitting rotation curve, solid line.

\subsection{2. $I Z w 18$}

One might expect mass loss of catastrophic proportion, since IZw18 is one of the least luminous galaxies yet contains a kiloparsec-scale polar bubble. (Note that only the southern lobe of the bipolar shell discussed by Martin (1996) was detected kinematically and therefore cataloged Table 3.) If the HI velocity field actually reflects a rotating disk in equilibrium, however, the dynamical mass within $r=850 \mathrm{pc}$ is $\sim 5 \times 10^{8} \mathrm{M}_{\odot}$ (Viallefond et al. 1987; van 
Zee et al. 1998) - a bit more than NGC 1569! In Figure 9, the projected expansion speed of the polar shell, $30 \mathrm{~km} \mathrm{~s}^{-1}$, is less than the escape speed from an $r_{0}=1.4 \mathrm{kpc}$ Burkert halo (dotted line). The deprojected shell velocity, closer to $\sim 90 \mathrm{~km} \mathrm{~s}^{-1}$ (Martin 1996), may exceed this escape velocity. The central density must be increased by 9.4 over Burkert's $\rho_{0}\left(r_{0}\right)$ relation to reach a circular velocity of $50 \mathrm{~km} \mathrm{~s}^{-1}$ (solid line). Since the dynamical mass estimate greatly exceeds the mass of this halo model, the argument for escape of the warm ionized shells is marginal.

\subsection{3. $N G C 1800$}

The shallow rise of the rotation curve interior to $r=6 \mathrm{kpc}$ is not entirely a resolution effect. In the inner part of NGC 1800, the HI velocity gradient rotates by $90^{\circ}$ and lies along the galaxy's minor axis (Hunter, van Woerden, \& Gallagher 1994). Hunter et al. (1994) suggested the gas is streaming along a stellar bar, and the ellipticity difference between the isophotes and isochromes confirm that the potential is non-axisymmetric (Quillen et al. 1996). The dynamical mass estimate is therefore uncertain since the gas is probably not on circular orbits in the inner few kiloparsecs, and the HI cloud about 2'4 west of the galaxy may not be in a circular orbit either. At a radius of $1.3 \mathrm{kpc}$ the escape velocity from an $r_{0}=1.4 \mathrm{kpc}$ halo, about $70 \mathrm{~km} \mathrm{~s}^{-1}$, is somewhat higher than the expansion speed of the northern polar bubble, $50 \mathrm{~km} \mathrm{~s}^{-1}$. Increasing the central halo density by a factor of 1.9 to fit the circular velocity at $r=4.7 \mathrm{kpc}$ raises the escape velocity to nearly $100 \mathrm{~km} \mathrm{~s}^{-1}$.

\subsection{4. $N G C 2366$ and $N G C 2363$}

NGC 2363 is a giant HII complex 90" southwest of the dynamical center of the dwarf galaxy NGC 2366 (e.g. Braun 1995). Since the distance of NGC 2363 from the center of the potential well, $R \sim 1.6 \mathrm{kpc}$, is greater than the radius of the bubbles, the shell velocities are plotted in Figure 9 against the radius of NGC 2366 rather than the shell radius. The expansion speed of shells A and B is less than the escape velocity, $\sim 145 \mathrm{~km} \mathrm{~s}^{-1}$, from an

$r_{0}=3.76 \mathrm{kpc}$ Burkert halo. This halo model provides a reasonably good description of the rotation curve, so the escape velocity is unlikely to be much higher. 


\subsubsection{NGC 3077}

The HI distribution in NGC 3077 is asymmetric. An HI stream extends northward from a large concentration in the southeast toward the outer spiral arm structure of M81 (Appleton et al. 1981; van der Hulst 1979). Despite this interaction, the velocity gradient along the optical major axis is surprisingly regular. The rotation curve in Figure 9 was estimated from the position - velocity diagram along the optical major axis (van der Hulst 1979) and corrected for an inclination of $38^{\circ}$. The maximum circular velocity at $r=6 \mathrm{kpc}$ is similar to the velocities of shells NGC 3077- A, J, B, and G, so the shells are traveling slower than the escape velocity. The bold, dashed line shows the escape velocity above the center of a disk with scalelength $3.76 \mathrm{kpc}$ and central surface density $133 \mathrm{M}_{\odot} \mathrm{pc}^{-2}$. The escape velocity from an $r_{0}=3.76 \mathrm{kpc}$ Burkert halo is similar. These models have a mass of $5.9 \times 10^{9} \mathrm{M}_{\odot}$ within $R\left(v_{\text {rot }}\right)=6.3 \mathrm{kpc}$. Higher resolution data might allow a smaller scalelength and lower escape velocity.

\subsection{6. $N G C 4861$}

The giant HII region shown in Figure 2 is about $1^{\prime}(2.18 \mathrm{kpc})$ southwest of the dynamical center of NGC 4861 (e.g. Wilcots et al. 1996b). The HI iso-velocity contours increase by $80 \mathrm{~km} \mathrm{~s}^{-1}$ from south to north over about 15 ! The circular velocity is then $\gtrsim 43 \mathrm{~km} \mathrm{~s}^{-1}$ at a radius $r \approx 16.5 \mathrm{kpc}$. The expansion speeds of shells $\mathrm{A}, \mathrm{B}$, and $\mathrm{C}$ are plotted against the distance of the giant HII region from the dynamical center of the galaxy in Figure 9. The escape velocity from a Burkert halo with core radius $r_{0}=2.31 \mathrm{kpc}$ is shown for comparison. The expansion speeds of shells A and B are of the same order as the circular velocity. The escape velocity at their location is probably a factor of several higher.

\subsection{7. $N G C 5253$}

The relatively high resolution $\left(\mathrm{FWHM} \approx 10^{\prime \prime}\right)$ HI map obtained by Kobulnicky (1997) shows a large disturbance in the radial velocity field southeast of NGC 5253. The velocity gradient along this half of the galaxy's minor axis is greater than that along the major axis. Similar gradients were measured in the $\mathrm{H} \alpha$ velocity field (Martin \& Kennicutt 1995). Hence, rotation does not dominate the velocity field of the interstellar gas in NGC 5253. The mass of visible gas and stars does place a lower limit on the dynamical mass of NGC 5253. For a composition $\mathrm{H} / \mathrm{He}=10 / 1$ by number, the mass of neutral gas is $\approx 1.96 \times 10^{8} \mathrm{M}_{\odot}$. The stellar mass is $\sim 2.3 \times 10^{8} \mathrm{M}_{\odot}$ for an assumed $\left(M / L_{B}\right)_{*}=1.0 \mathrm{M}_{\odot} / \mathrm{L}_{\odot}$. The Keplerian 
velocity at the extent of the detected HI, $r=1.24 \mathrm{kpc}$, would then be at least $38 \mathrm{~km} \mathrm{~s}^{-1}$. If the mass is centrally concentrated, then the circular velocity at $r=500 \mathrm{pc}$, the edge of the $\mathrm{H} \alpha$ nebula, could be as high as $60 \mathrm{~km} \mathrm{~s}^{-1}$. The estimated escape velocity is at least a factor of 1.4 times higher. The swept-up shell expanding at $\sim 35 \mathrm{~km} \mathrm{~s}^{-1}$ is then marginally bound to NGC 5253 .

\subsubsection{NGC 4214}

The HI rotation axis in NGC 4214 is nearly aligned with the optical major axis; and McIntyre (1996) suggests the strong central iso-velocity twist could be the signature of a bar. The extended disk of HI in NGC 4214 reaches a circular rotation speed of $\sim 70 \mathrm{~km} \mathrm{~s}^{-1}$ at a radius $R=2.91 \mathrm{kpc}$ (McIntyre 1996). Like NGC 3077, much of the mass in the inner region of NGC 4214 could, however, be provided by a disk of stars and gas. The bold, dashed line in the figures shows that the escape velocity above the center of a disk with central surface density $235 \mathrm{M}_{\odot} / \mathrm{pc}^{2}$ and scalelength $r_{0}=2.91 \mathrm{kpc}$. The escape velocity from an

$r_{0}=2.91 \mathrm{kpc}$ halo model is lower, but that from a denser halo that dominates the interior mass would be higher. Both estimates of the halo escape velocity one kiloparsec from the starburst are somewhat higher than the shell speeds which range from $28-100 \mathrm{~km} \mathrm{~s}^{-1}$.

\subsection{9. $N G C 4449$}

The HI surrounding NGC 4449 extends about 14 times farther than the optical galaxy. The gravitational interaction with DDO 125 is probably responsible for some distortion in the velocity field (Bajaja, Huchtmeier, \& Klein 1994), but the disk seems to be in regular rotation about the center of NGC 4449 (Wilcots et al. 1996a). The velocity increases from north to south across the extended disk - a gradient opposite to that observed across the inner few arcminutes (e.g. see echellograms NGC 4449-1, 2, 7, and 10). The rotation curve in Figure 9 is based on the position - velocity diagram that Bajaja et al. extracted along the HI ridgeline. The velocity rises to $65 \mathrm{~km} \mathrm{~s}^{-1} 15.7 \mathrm{kpc}$ from the galactic center. For an inclination of $51^{\circ}$, the maximum circular velocity is then $84 \mathrm{~km} \mathrm{~s}^{-1}$ which is near the middle of the range of shell expansion speeds $\left(30-150 \mathrm{~km} \mathrm{~s}^{-1}\right)$. For comparison, the circular velocity of a halo with core radius $r_{0}=6.3 \mathrm{kpc}$ is shown in Figure 9. If a spherically symmetric halo of this type is a good description of the gravitational potential in NGC 4449, then the gas swept into the expanding shells is bound to NGC 4449. 


\subsubsection{M82}

The last panel Figure 9 shows a rotation curve derived from measurements of stellar absorption lines along the major axis of M82 (Burbidge, Burbidge, \& Rubin 1964). The light and bold dotted lines show the circular velocity and escape velocity, respectively, from an $r_{0}=3.76 \mathrm{kpc}$ Burkert halo. Increasing the central density by a factor of 22.1 over Burkert's $\rho_{0}\left(r_{0}\right)$ relation to raise the interior halo mass to the estimated dynamical mass raises the upper limit on the escape velocity from $130 \mathrm{~km} \mathrm{~s}^{-1}$ to $650 \mathrm{~km} \mathrm{~s}^{-1}$. Although this outflow is clearly in a differenct class energetically, it is not clear that even this warm ionized gas will escape the deeper potential.

\subsection{Conclusions}

\subsubsection{Galatic Mass Loss and Blow Away}

The circular velocities of the program galaxies are compiled in Table 1 for reference. They span a factor of 4 in magnitude - a range similar to the velocity distribution of kiloparsec-scale shells. The observed mass of stars and gas alone place a substantial lower limit on the escape velocity in several galaxies. Halos with core radii that reproduce the turnovers in the rotation curves predict even larger escape velocities since they extend to $\sim 160 \mathrm{kpc}$. The best estimates of the escape speeds from the current data are typically a factor of 1.5 to 3 times higher than the projected expansion velocity. However, while the potential does appear to be relatively deep in some of the galaxies with the most energetic outflows (e.g. M 82 and NGC 4449), large and/or fast shells are also found in some less luminous galaxies. In the case of Sex A, and quite possibly I $\mathrm{Zw}_{\mathrm{w}} 18$, the shell velocities are comparable to $v_{e s c}$. Substantial loss of shell material seems most likely in NGC 1569 where mutiple shells have propagated well above the galactic plane, but the galaxy is apparently not very massive.

In contrast to the warm ioninzed gas in the shells, the hot, X-ray emitting gas at high latitudes is not bound to the galaxy. An escape velocity of $100 \mathrm{~km} \mathrm{~s}^{-1}$, for example, is equivalent to an escape temperature of $4 \times 10^{5} \mathrm{~K}$ (e.g. Wang 1995; Martin 1996). The soft X-ray halos detected around NGC 1569, NGC 4449, and M82 have X-ray temperatures of

$\sim 7-9 \times 10^{6} \mathrm{~K}$ (Della Ceca et al. 1996; Della Ceca et al. 1997; Strickland et al. 1997). The position angles of the X-ray lobes protruding from these galaxies correlate strongly with those of the extended $\mathrm{H} \alpha$ filaments, so the lobes of hot gas are probably at least partially confined by cooler surrounding gas. This hot gas will escape from the galaxy in a wind if the confining shell breaks up. Estimates of the mass in the hot ISM are compiled in Table 5 
for a volume filling factor $\sim 1$. The mass in hot gas is only a few percent, of the interstellar HI mass in the two dwarfs galaxies where it is measured.

The current star formation rates in these galaxies are at least an order of magnitude too low to instantly (i.e. $t<10^{7} \mathrm{yr}$ ) expel the ISM. For each galaxy with a dynamical mass estimate, the total kinetic energy in large-scale shells was compared to a lower limit on the gravitational binding energy of the interstellar gas, $E_{B} \gtrsim\left(1.4 M_{H I}\right) v_{\text {circ }}^{2}$. Their ratio, column 11 of Table 5, is less than one for all the galaxies. The NGC 1569 outflow has the highest ratio, K.E./ $E_{B} \lesssim 0.3$. As emphasized by DeYoung \& Heckman (1994), the transfer of this energy, which is largely in polar outflows, to the ambient disk may be quite inefficient. Hence, if the star formation rate is rapidly declining in these galaxies, then the bulk of their interstellar gas will remain in the disk.

\subsubsection{Disk Mass Loss}

These data demonstrate that superbubbles lift significant masses of gas out of the star-forming disk. Regardless of whether any shell material actually escapes from the galaxy, the efficiency of this re-heating is an important parameter in models of galaxy formation and evolution. Table 5 summarizes the upper limits on the disk mass loss rates. These rates represent the transfer of warm ionized gas and hot ionized gas from the star-forming disk to the halo and were calculated by dividing the mass in ionized shells and hot gas by the dynamical age of the oldest polar bubble. Although measurements of the filling factor $\epsilon$ may lower the mass loss rates by factors of a few, the disk mass loss rates will likely remain significant as they are typically slightly greater than the current star formation rates.

If star forming regions similar in scale to the current giant HII regions continue to form, the mass blown into the halo of several of the galaxies will be significant. The timescale for removing the ISM is of order the total gas mass, $\sim 1.4 M_{H I}$, divided by the estimated mass loss rates in Table 5. These values range from $50 \mathrm{Myr}$ and $140 \mathrm{Myr}$ for M82 and NGC 1569, respectively, to 11 Gyr for NGC 4214. In Table 5, I compare these timescales to the rotational period of the disk. The rotation period was computed at the radius where the circular speed was tabulated and is representative of the inner disk which shows solid body since rotation. Associating this period with a timescale for star formation, $\tau_{*}$, the bulk of the HI disk could be removed in less than $6 \tau_{*}$ for the following galaxies: NGC 1569, NGC 1800, M82, NGC 3077, NGC 4449, and NGC 4861. 


\section{Summary}

An extensive set of $\mathrm{H} \alpha$ echellograms and images were used to reconstruct the large-scale kinematics of the ionized gas in 14 dwarf galaxies and M82. Details of the results for individual galaxies are included in their respective subsections of the paper, and Figure 1 provides a concise summary of the shell expansion speeds and sizes. The main results regarding the formation of winds in dwarf galaxies are summarized here.

- The formation of supershells must be a common byproduct of massive star formation in dwarf galaxies. Expanding, supergiant $(R>300 \mathrm{pc})$ shells were found in all but two of the galaxies. This sample was drawn from the population of nearby dwarf galaxies with prominent arcs and/or extended filaments in their $\mathrm{H} \alpha$ emission, and roughly one out of four catalogued dwarf galaxies fits this description (Hunter, Hawley, \& Gallagher 1993). Indeed, the hierarchical growth of these structures probably began in star forming regions akin to 30 Doradus in the Large Magellanic Cloud (Chu \& Kennicut 1994). The most powerful outflows, i.e. NGC 1569 and M82, were found to be composed of multiple cells whose walls are probably the interface between polar shells. Star formation in the lowest luminosity galaxies, e.g. IZw18, also generates kiloparsec-scale shells.

- Although many of the expanding complexes survive for $10 \mathrm{Myr}$, none older than $\sim 20$ Myr were identified. The lack of shells older than this likely reflects their disruption timescale and provides an indirect measure of the scale height of the ISM. Alternatively, the ionization rate of the shells might drop abruptly on this timescale due to changes in the birthrate of massive stars and/or the illumination geometry. Although bright, extraplanar HI shells have not been detected in any of the galaxies in this sample, some HI holes in the LMC (Meaburn 1980) and NGC 4449 (Hunter \& Gallagher 1997) are probably relics of expanding supershells. The power requirements of the ionized supershells typically exceed the critical power for supersonic disk breakthrough, so a disruption scenario must be favored for them. The sequence of echellograms stepped across the southern lobe of NGC 1569 constrains their deceleration and shows multiple velocity components at least up to 640 pc above the galactic plane. Future observational work must aim to detect the remains of the hot gas and ruptured shell following blowout. One might speculate that the quiescent filaments in NGC 5253 could be fragments of a ruptured shell or clumps of infalling material ejected in a previous wind epoch. A better census of the local dwarf population would also be helpful for constraining the duty cycle of the winds.

- Presuming the shells do rupture, the escape of hot, X-ray emitting gas from their 
interiors seems certain. A diffuse, thermal component of the X-ray emission has been resolved in three of the galaxies in the sample, but it is only a significant fraction $(>10 \%)$ of the interstellar HI mass in M82. In contrast, much of the interstellar gas swept into the warm ionized shells probably remains bound to the galaxy. The structure of the dark matter halo has been measured in several low surface brightness dwarf galaxies with large HI disks (e.g. Carignan \& Beaulieu 1989; Meurer et al. 1994) and appears to have a universal structure (Burkert 1995; Navarro, Eke, \& Frenk 1996). Hence, a conservative approach to mass loss is to assume that the bursting dwarfs are embedded in similar halos. These dark halo models often do not provide enough mass to explain the HI rotation speed in the inner galaxy, however. Stars and atomic gas can account for essentially all the dynamical mass inside $R\left(v_{\text {circ }}\right)$ in some galaxies like NGC 1569, but the fraction varies enormously among the sample members. Although little CO emission is detected from the dwarf galaxies (e.g. Young et al. 1996), the large uncertainty in the $H_{2}$ to CO conversion factor does allow substantial mass contributions from molecular gas (e.g. Maloney \& Black 1988). A dominant disk in the inner few kiloparsecs has two immediate implications. First, the disk mass contributes significantly to the gravitational acceleration of the kiloparsec-scale shells/disk outflows. Second, the observed turnover in the rotation curve may not be revealing much about the core radius of the halo - a critical parameter for estimating the escape velocity. The maximum circular velocities in the galaxies do generally appear to be comparable to the expansion velocities of the supershells, so the escape velocities are greater than the projected shell speed. The expansion speeds along the minor axis of NGC 1569 do reach values several times the maximum rotation speed.

- The warm shells alone lift gas out of the disk at rates comparable to or greater than the current galactic star formation rates. The shells transport $10^{5}$ to $10^{6} \mathrm{M}_{\odot}$ of gas over kiloparsec-scale shells in $10 \mathrm{Myr}$ and leave the sound speed high in a large volume of the ISM. This hydrodynamic mixing will be faster than a diffusion process, so the bubbles will clearly alter the chemical evolution of these galaxies. The timescales for blowout are shorter than the evolutionary timescales of most models for Type Ia supernova progenitors, so the mass loss begins before much of the iron from the burst has been mixed into the ISM. The composition of the ejected material will depend on the duration of the wind and the composition of the ambient ISM, so their impact on the galactic chemical evolution is interwoven with the galactic star formation history.

- Although the current kinetic energy in the large expanding structures is only comparable to the binding energy of the ISM in NGC 1569, bubble blowout may still extinguish star formation in particular regions of the other galaxies. If the hot spots percolate across the dwarf irregular galaxies for many rotational periods, then a 
substantial fraction of the interstellar gas may still be cycled through a halo and/or lost from the galaxy. If the present mass loss rates could be sustained for 6 orbital timescales, for example, then most of the interstellar HI could be removed from the disks of six of the 15 galaxies. This global gas-dynamical feedback will be discussed in the context of the galactic star formation history in a forthcoming paper.

I would like to thank Chip Kobulnicky, Vince McIntyre, and Eric Wilcots for providing results from HI observations prior to publication. I am grateful to Rob Kennicutt and Tim Heckman for their comments and suggestions about the manuscript, and to Rob Kennicutt for assisting with some of the initial data acquisition. Financial support was provided by an NSF Graduate Fellowship, Hubble Fellowship grant HF-01083.01-96A, and NSF grant AST-9421145. 
Table 1: The Sample of Galaxies

\begin{tabular}{lllllllll}
\hline Galaxy & $\begin{array}{l}\mathrm{d}^{\mathrm{a}} \\
(\mathrm{Mpc})\end{array}$ & $\begin{array}{l}M_{B}{ }^{\mathrm{b}} \\
(\mathrm{mag})\end{array}$ & $\begin{array}{l}\mathrm{D}^{\mathrm{c}} \\
\left.{ }^{\prime}\right)\end{array}$ & $\begin{array}{l}\text { HI Extent } \\
(\mathrm{kpc} \times \mathrm{kpc})\end{array}$ & $\begin{array}{l}v_{\text {circ }}^{\text {proj }} \\
(\mathrm{km} / \mathrm{s})\end{array}$ & $\begin{array}{l}v_{\text {circ }} \mathrm{f}^{\mathrm{f}} \\
(\mathrm{km} / \mathrm{s})\end{array}$ & $\begin{array}{l}R\left(v_{\text {circ }}\right)^{\mathrm{g}} \\
(\mathrm{kpc})\end{array}$ & HI Ref. $^{\mathrm{h}}$ \\
\hline VII Zw 403 & 3.6 & -13.68 & 3.6 & $\cdots$ & $\cdots$ & $\cdots$ & $\ldots$ & 1 \\
Sextans A & 1.31 & -13.84 & 1.31 & $3.4 \times 3.4$ & 20 & 34 & 1.2 & 2,3 \\
I Zw 18 & 10.0 & -13.84 & 0.5 & $2.9 \times 1.5$ & 50 & $\ldots$ & 0.853 & 4,5 \\
II Zw 40 & 10.1 & -14.54 & 1.3 & $15.2 \times 2.4$ & 53 & $\ldots$ & 5.2 & 6,7 \\
NGC 3738 & 4.0 & -16.0 & 4.0 & $\ldots$ & $\ldots$ & $\ldots$ & $\ldots$ & 8 \\
NGC 1800 & 8.1 & -16.67 & 2.2 & $13.9 \times 8.08$ & 40 & 46 & 5.7 & 9,10 \\
NGC 2363 & 3.6 & -16.75 & 8.0 & $7.67 \times 16.7$ & 53 & 63 & 5.5 & 6,11 \\
NGC 1569 & 2.2 & -17.26 & 3.1 & $4.2 \times 3.2$ & 28 & 31 & 1.0 & 12,13 \\
NGC 2537 & 7.5 & -17.36 & 1.7 & $\ldots$ & $\ldots$ & $\ldots$ & $\ldots$ & 6 \\
NGC 3077 & 3.6 & -17.37 & 3.6 & $12.7 \times 8.0$ & 40 & 65 & 6.3 & 14,15 \\
NGC 4861 & 7.5 & -17.6 & 7.5 & $11.9 \times 15.7$ & 40 & 43 & 16.5 & 16 \\
NGC 5253 & 4.1 & -17.62 & 4.1 & $4.3 \times 4.3$ & $<15$ & $38-60$ & 1.24 & $17,18,19$ \\
NGC 4214 & 3.6 & -17.65 & 3.6 & $14.6 \times 12.9$ & 35 & 70 & 2.91 & 20,21 \\
NGC 4449 & 3.6 & -17.86 & 3.6 & $43 \times 70$ & 65 & 84 & 15.7 & 22 \\
M 82 & 3.6 & -18.95 & 3.6 & $4.19 \times 1.57$ & 135 & 136 & 2.0 & $14,23,24$ \\
\hline
\end{tabular}

${ }^{a}$ (col. 2) Adopted distance.

${ }^{b}$ (col. 3) Absolute blue magnitude from the RC3 blue magnitude (deVaucouleurs 1991).

${ }^{c}$ (col. 4) Angular diameter at the 25 B-magnitudes per square arcsecond isophote from Tully (1988).

${ }^{d}$ (col 5) Spatial dimensions of the neutral hydrogen.

e(col 6) Projected rotation speed.

${ }^{f}$ (col 7) Rotation speed corrected for the disk inclination when known.

$g$ (col 8) Radius at which the rotation speed in col. 6 was measured.

${ }^{h}$ References: (1) Tully, Boesgaard, Dyck, \& Schempp 1981. (2) Skillman, Terlevich, Teuben, \& van Woerden 1988. (3) Huchtmeier, Seiradakis, \& Materne 1981. (4) Viallefonde, Lequeux, \& Comte 1987. (5) Lequeux, \& Viallefond 1980. (6) Thuan, \& Martin 1981. (7) Brinks, \& Klein 1988. (8) Hunter, Gallagher, \& Rautenkranz 1982. (9) Hunter, van Woerden, \& Gallagher 1994. (10) Gallagher, Knapp, \& Hunter 1981. (11) Braun 1995. (12) Israel \& van Driel 1990. (13) Reakes 1980. (14) Appleton, Davies, \& Stephenson 1981. (15) van der Hulst 1979. (16) Wilcots, Lehman, \& Miller 1996. (17) Kobulnicky, \& Skillman 1995. (18) Reif, Mobold, Goss, vanWoerden, \& Siegman 1982. (19) Kobulnicky 1997. (20) Kobulnicky \& Skillman 1996. (21) McIntyre 1997. (22) Bajaja, Huchtmeier, \& Klein 1994. (23) Burbidge, Burbidge, \& Rubin 1964. (24) Yun, Ho, \& Lo 1993. 
Table 2: Observations

\begin{tabular}{|c|c|c|c|c|}
\hline Telescope & Instrument/Detector & Date & Filter/Grating & Conditions \\
\hline $\begin{array}{l}\text { Steward } 2.3 \mathrm{~m} \\
\text { " }\end{array}$ & $\begin{array}{l}\text { Cass Camera + Loral } 800 \times 1200 \text { CCD } \\
"\end{array}$ & $\begin{array}{l}1993 \text { April } 21 \text { and } 28 \\
1993 \text { May } 16-17\end{array}$ & $\underset{\text { " }}{\lambda} 6450, \lambda 6580,70 \AA \mathrm{FWHM}$ & Clear \\
\hline " & $"$ & 1994 January 3 & " & " \\
\hline “ & Cass Camera $+2 \mathrm{k} \times 2 \mathrm{k}$ CCD & 1993 December 5-6 & “ & Variable \\
\hline " & Focal reducer + TI $800 \times 800$ & 1990 March 22 & $"$ & \\
\hline KPNO $4 \mathrm{~m}$ & $\begin{array}{l}\text { Echelle }+ \text { Red Long }+ \text { T2KB } \\
\text { ( }\end{array}$ & $\begin{array}{l}1994 \text { April } 28 \text { - May } 2 \\
1995 \text { Dec } 10-11,1996\end{array}$ & $\underset{\text { " }}{\mathrm{H} \alpha} 6563 \mathrm{~B}, 79-63^{\circ}$ & $\begin{array}{l}\text { Good } \\
\text { Variable }\end{array}$ \\
\hline “ & “ & February $7-8$ & " & " \\
\hline
\end{tabular}


Table 3: Properties of Supershells

\begin{tabular}{|c|c|c|c|c|c|c|c|c|c|c|}
\hline Complex ${ }^{a}$ & Slits b & $\begin{array}{r}\mathrm{v}^{\mathrm{c}} \\
(\mathrm{km} / \mathrm{s})^{-}\end{array}$ & $\begin{array}{l}\mathrm{R}^{\mathrm{d}} \\
\left({ }^{\prime \prime}\right)\end{array}$ & $\begin{array}{r}R \\
(\mathrm{pc})\end{array}$ & $\begin{array}{c}\text { Aperture } \\
\quad\left(\operatorname{asec}^{2}\right)\end{array}$ & $\Sigma_{\mathrm{H} \alpha+[N I I]} \mathrm{f}$ & $\begin{array}{c}M \sqrt{\epsilon / 0.1} \mathrm{~g} \\
\left(10^{6} \mathrm{M}_{\odot}\right)\end{array}$ & $\begin{array}{c}\mathrm{KE} \sqrt{\epsilon / 0.1^{\mathrm{h}}} \\
\left(10^{51} \text { ergs }\right)\end{array}$ & $(\mathrm{Myr})$ & $\begin{array}{c}L_{i n}(1) / \bar{n}(1)^{\mathrm{j}} \\
\left(\mathrm{cm}^{3} \mathrm{ergs} / \mathrm{s}\right)\end{array}$ \\
\hline SexA-A & 1 & 62 & 13.9 & 88 & $7.209 \mathrm{e} 2$ & $2.38 \mathrm{e}-16$ & 0.027 & 1.0 & 0.83 & $7.3 \mathrm{e} 38$ \\
\hline $\mathrm{IZw} 18-\mathrm{S}$ & 1 & 34 & 19.9 & 970 & $1.620 \mathrm{e} 2$ & $1.98 \mathrm{e}-16$ & 0.47 & 5.4 & 17 & $1.5 \mathrm{e} 40$ \\
\hline NGC3738-A & 2,3 & 35 & 8.9 & 173 & $1.878 \mathrm{e} 2$ & $1.58 \mathrm{e}-15$ & 0.060 & 0.73 & 2.9 & $5.1 \mathrm{e} 38$ \\
\hline B & 2 & 24 & 6.8 & 132 & $9.027 \mathrm{e} 1$ & $2.43 \mathrm{e}-16$ & 0.015 & 0.088 & 3.2 & $9.6 \mathrm{e} 37$ \\
\hline $\mathrm{C}$ & 2 & 17 & 14.9 & 288 & $2.535 \mathrm{e} 2$ & $9.47 \mathrm{e}-16$ & 0.13 & 0.38 & 9.9 & $1.6 \mathrm{e} 38$ \\
\hline NGC1800-N & $2, \mathrm{PA} 210^{\circ}$ & $50^{k}$ & 34.3 & 1350 & $1.497 \mathrm{e} 3$ & $6.16 \mathrm{e}-17$ & 2.0 & 49 & 16 & $9.1 \mathrm{e} 40$ \\
\hline NGC2363-A & $1,5,6$ & 44 & 34 & 593 & $1.533 \mathrm{e} 2$ & $9.68 \mathrm{e}-17$ & 0.76 & 15 & 7.9 & $1.2 \mathrm{e} 40$ \\
\hline B & 5 & 69 & 38.4 & 670 & $1.023 \mathrm{e} 2$ & $3.66 \mathrm{e}-16$ & 1.04 & 49 & 5.7 & $5.9 \mathrm{e} 40$ \\
\hline NGC1569-C & 10,11 & 79 & 8.07 & 85 & $2.096 \mathrm{e} 2$ & $1.45 \mathrm{e}-14$ & 0.23 & 14 & 0.6 & $1.4 \mathrm{e} 39$ \\
\hline $\mathrm{A}$ & $10,13,4,7$ & 120 & 105 & 1120 & $3.960 \mathrm{e} 3$ & $4.69 \mathrm{e}-16$ & 1.7 & 250 & 5.5 & $8.6 \mathrm{e} 41$ \\
\hline B & 11,6 & 85 & 90.3 & 960 & $4.451 \mathrm{e} 3$ & $3.13 \mathrm{e}-16$ & 1.6 & 120 & 6.6 & $2.2 \mathrm{e} 41$ \\
\hline $\mathrm{D}$ & 10,6 & 54 & 98.4 & 1050 & $2.651 \mathrm{e} 3$ & $4.66 \mathrm{e}-16$ & 0.84 & 24 & 11 & $6.9 \mathrm{e} 40$ \\
\hline $\mathrm{E}$ & 12,6 & 44 & 76.6 & 820 & $3.410 \mathrm{e} 3$ & $2.48 \mathrm{e}-16$ & 1.1 & 20 & 11 & $2.3 \mathrm{e} 40$ \\
\hline $\mathrm{F}$ & $4,13,7$ & 64 & 83.8 & 890 & $3.035 \mathrm{e} 3$ & $4.61 \mathrm{e}-16$ & 2.2 & 89 & 8 & $8.2 \mathrm{e} 40$ \\
\hline G & $4,7,11,13$ & 64 & 99.5 & 1060 & $2.736 \mathrm{e} 3$ & $3.66 \mathrm{e}-16$ & 1.2 & 49 & 9.7 & $1.2 \mathrm{e} 41$ \\
\hline NGC2537-A & 1,3 & 47 & 4.8 & 175 & $1.095 \mathrm{e} 2$ & $8.42 \mathrm{e}-17$ & 0.087 & 1.9 & 2.2 & $1.3 \mathrm{e} 39$ \\
\hline big & & 47 & 22.1 & 800 & $3.118 \mathrm{e} 2$ & $2.96 \mathrm{e}-17$ & 0.10 & 2.3 & 10 & $2.6 \mathrm{e} 40$ \\
\hline NGC3077-D & 3,2 & 106 & 17.7 & 310 & $3.263 \mathrm{e} 2$ & $3.29 \mathrm{e}-15$ & 0.32 & 35 & 1.7 & $4.5 \mathrm{e} 40$ \\
\hline $\mathrm{A}$ & 5 & 55 & 46.2 & 810 & $1.298 \mathrm{e} 3$ & $5.71 \mathrm{e}-16$ & 1.4 & 43 & 8.6 & $4.3 \mathrm{e} 40$ \\
\hline $\mathrm{J}$ & 2,4 & 67 & 53.8 & 940 & $1.342 \mathrm{e} 3$ & $2.91 \mathrm{e}-16$ & 1.0 & 45 & 8.2 & $1.1 \mathrm{e} 41$ \\
\hline B & $5,2,3$ & 40 & 36.9 & 640 & $6.256 \mathrm{e} 2$ & $1.23 \mathrm{e}-15$ & 0.93 & 15 & 9.4 & $1.0 \mathrm{e} 40$ \\
\hline G & 2 & 51 & 47.8 & 830 & $9.076 \mathrm{e} 2$ & $4.06 \mathrm{e}-16$ & 0.61 & 16 & 10 & $3.6 \mathrm{e} 40$ \\
\hline NGC4861-A & 1,4 & 50 & 23.4 & 850 & $5.024 \mathrm{e} 2$ & $3.9 \mathrm{e}-16$ & 2.7 & 66 & 10 & $3.6 \mathrm{e} 40$ \\
\hline $\mathrm{C}$ & 2,4 & 30 & 26.4 & 960 & $2.894 \mathrm{e} 2$ & $3.8 \mathrm{e}-16$ & 1.3 & 12 & 19 & $9.9 \mathrm{e} 39$ \\
\hline B & 1 & 43 & 15.7 & 570 & $3.065 \mathrm{e} 2$ & $7.8 \mathrm{e}-16$ & 1.2 & 23 & 7.8 & $1.0 \mathrm{e} 40$ \\
\hline NGC5253-E & $\mathrm{PA} 60^{\circ}$ & $35^{k}$ & 43.8 & 870 & $1.935 \mathrm{e} 3$ & $2.37 \mathrm{e}-16$ & 1.2 & 14 & 15 & $1.3 \mathrm{e} 40$ \\
\hline NGC4214-A & $1,2,3$ & 45 & 8.33 & 150 & $1.350 \mathrm{e} 2$ & $1.27 \mathrm{e}-14$ & 0.13 & 2.8 & 2 & $7.6 \mathrm{e} 38$ \\
\hline B & 1 & 28 & 30.3 & 530 & $6.698 \mathrm{e} 2$ & $1.69 \mathrm{e}-15$ & 1.1 & 8.3 & 11 & $2.4 \mathrm{e} 39$ \\
\hline $\mathrm{C}$ & 1 & 100 & 30.7 & 540 & $2.597 \mathrm{e} 2$ & $1.13 \mathrm{e}-15$ & 0.41 & 41 & 3.2 & $1.1 \mathrm{e} 41$ \\
\hline $\mathrm{D}$ & 4 & 46 & 14.2 & 250 & $6.597 \mathrm{e} 1$ & $3.58 \mathrm{e}-15$ & 0.14 & 3.0 & 3.2 & $2.4 \mathrm{e} 39$ \\
\hline $\mathrm{F}$ & 2 & 44 & 37.4 & 630 & $6.869 \mathrm{e} 2$ & $1.01 \mathrm{e}-15$ & 0.61 & 12 & 8.4 & $1.3 \mathrm{e} 40$ \\
\hline NGC4449-A & $1,2,3$ & 79 & 43.1 & 950 & $1.440 \mathrm{e} 3$ & $5.82 \mathrm{e}-16$ & 1.5 & 95 & 7.1 & $1.8 \mathrm{e} 41$ \\
\hline $\mathrm{C}$ & $1,2,3,11$ & 30 & 26.8 & 470 & $2.163 \mathrm{e} 3$ & $9.49 \mathrm{e}-16$ & 3.4 & 31 & 9.2 & $2.4 \mathrm{e} 39$ \\
\hline D & 9,11 & 33 & 13.6 & 240 & $7.295 \mathrm{e} 2$ & $5.44 \mathrm{e}-16$ & 0.49 & 5.3 & 4.3 & $8.2 \mathrm{e} 38$ \\
\hline G & 9 & 25 & 14.4 & 250 & $1.642 \mathrm{e} 3$ & $8.12 \mathrm{e}-16$ & 1.7 & 11 & 5.9 & $3.9 \mathrm{e} 38$ \\
\hline $\mathrm{H}$ & 7 & 51 & 43.9 & 770 & $1.045 \mathrm{e} 3$ & $4.92 \mathrm{e}-16$ & 1.0 & 27 & 8.9 & $3.1 \mathrm{e} 40$ \\
\hline I & 7 & 50 & 25.7 & 450 & $1.067 \mathrm{e} 3$ & $1.25 \mathrm{e}-15$ & 1.4 & 35 & 5.3 & $1.0 \mathrm{e} 40$ \\
\hline E4 & 10 & 27 & 3.4 & 60 & $4.473 \mathrm{e} 1$ & $5.04 \mathrm{e}-16$ & 0.012 & 0.090 & 1.3 & $2.8 \mathrm{e} 37$ \\
\hline $\mathrm{E} 2$ & 7 & 31 & 7.2 & 126 & $2.702 \mathrm{e} 2$ & $8.08 \mathrm{e}-16$ & 0.090 & 0.86 & 2.4 & $1.9 \mathrm{e} 38$ \\
\hline E3 & 10 & 146 & 7.7 & 130 & $2.654 \mathrm{e} 2$ & $8.49 \mathrm{e}-16$ & 0.14 & 31 & 0.5 & $2.1 \mathrm{e} 40$ \\
\hline E1 & 10 & 40 & 4.5 & 79 & $2.158 \mathrm{e} 2$ & $3.07 \mathrm{e}-16$ & 0.060 & 0.96 & 1.2 & $1.6 \mathrm{e} 38$ \\
\hline M82-N & 2,4 & 156 & 96.2 & 1679 & $3.681 \mathrm{e} 4$ & $4.28 \mathrm{e}-16$ & 55 & 13400 & 6.3 & $4.2 \mathrm{e} 42$ \\
\hline $\mathrm{S}$ & 2 & 135 & 126.7 & 2210 & $1.999 \mathrm{e} 4$ & $6.32 \mathrm{e}-16$ & 29 & 5300 & 9.6 & $4.8 \mathrm{e} 42$ \\
\hline
\end{tabular}

${ }^{a}$ (col. 1) Name given to each expanding complex of warm, ionized filaments (see Figure 2).

${ }^{b}$ (col. 2) Slits along which the complex was kinematically detected.

${ }^{c}$ (col. 3) Maximum expansion velocity along our sightline.

${ }^{d}$ (cols. 4, 5) Projected distance of shell from star-forming region.

${ }^{e}$ (col. 6) Area of aperture in Figure 2.

${ }^{f}($ col. 7$)$ Surface brightness $(\mathrm{H} \alpha+[\mathrm{NII}])$ of emission-line filaments within the polygonal apertures shown in Figure 2. Units are ergs $/ \mathrm{s} / \mathrm{cm}^{2} / \mathrm{asec}^{2}$.

${ }^{g}$ (col. 8) Upper limit on mass of warm ionized gas in the expanding complex, where $M=14 / 11 n_{r m s} m_{\mathrm{H}} V \epsilon^{1 / 2}$. Warm ionized clouds are assumed to fill a fraction $\epsilon=0.1$ of the bubble volume, $V$; and the root mean square electron density is derived from $\mathrm{H} \alpha$ photometry (see $\S 4.2$.

${ }^{h}$ (col. 9) Estimate of kinetic energy in expanding shell, $K E=0.5 M v^{2}$.

${ }^{i}$ (col. 10) Dynamical age of shell, $\tau=0.6 R / v$.

${ }^{j}$ (col. 11) Dynamical estimate of kinetic energy injection rate (see $\left.\S 4.3\right)$. The inferred power scales linearly with the average ambient hydrogen density $\bar{n}(1)$.

${ }^{k}$ Measurement from Marlowe et al. (1995). 
Table 4: Global Star Formation Rates

\begin{tabular}{llllll}
\hline \hline Galaxy & $\begin{array}{l}\mathrm{F}(\mathrm{H} \alpha+[\mathrm{NII}])^{\mathrm{a}} \\
\left(\mathrm{ergs} / \mathrm{s} / \mathrm{cm}^{2}\right)\end{array}$ & $\begin{array}{l}\mathrm{c}(\mathrm{H} \beta)^{\mathrm{b}} \\
(\mathrm{mag})\end{array}$ & $\begin{array}{l}{[\mathrm{NII}] / \mathrm{H} \alpha} \\
\end{array}$ & $\begin{array}{l}\mathrm{Q}^{\mathrm{d}} \\
\left(\mathrm{s}^{-1}\right)\end{array}$ & $\begin{array}{l}\mathrm{M}^{\mathrm{e}} \\
\mathrm{M}_{\odot} \mathrm{yr}^{-1}\end{array}$ \\
\hline VII Zw 403 & $5.94 \pm 0.2 e-13$ & 0.1 & 0.027 & $7.68 \mathrm{e} 50$ & 0.0024 \\
Sex A-EW & $>2.44 \pm 0.55 e-12^{\mathrm{f}}$ & 0.021 & 0.021 & $6.58 \mathrm{e} 50 \mathrm{~g}$ & 0.0020 \\
I Zw 18 & $4.44 \pm 0.1 e-13$ & 0 & 0.004 & $3.87 \mathrm{e} 51$ & 0.012 \\
II Zw 40 & $2.23 \pm 0.90 e-12$ & 1.1 & 0.026 & $1.05 \mathrm{e} 53$ & 0.33 \\
NGC 3738 & $1.40 \pm 0.05 e-12$ & 0.5 & 0.063 & $3.97 \mathrm{e} 51$ & 0.013 \\
NGC 1800 & $6.30 \pm 2.0 e-13$ & 1.0 & 0.150 & $1.45 \mathrm{e} 52$ & 0.046 \\
NGC 2363 & $5.04 \pm 0.2 e-12$ & 0.1 & 0.026 & $6.49 \mathrm{e} 51$ & 0.021 \\
NGC 1569 & $2.31 \pm 1.1 e-11$ & 0.83 & 0.025 & $3.41 \mathrm{e} 52$ & 0.11 \\
NGC 2537 & $7.98 \pm 0.83 e-14$ & 0.3 & 0.160 & $5.37 \mathrm{e} 50$ & 0.0017 \\
NGC 3077 & $1.74 \pm 0.08 e-11$ & 0.8 & 0.256 & $5.36 \mathrm{e} 52$ & 0.170 \\
NGC 4861 & $2.75 \pm 0.4 e-12$ & 0.07 & 0.019 & $1.48 \mathrm{e} 52$ & 0.047 \\
NGC 5253 & $1.53 e-11$ & 0.4 & 0.077 & $3.86 \mathrm{e} 52$ & 0.12 \\
NGC 4214 & $1.47 \pm 0.16 e-11$ & 0.14 & 0.062 & $1.94 \mathrm{e} 52$ & 0.061 \\
NGC 4449 & $2.42 \pm 1.7 e-11$ & 0.27 & 0.127 & $3.68 \mathrm{e} 52$ & 0.12 \\
\hline
\end{tabular}

${ }^{a}$ (col. 2) Total $\mathrm{H} \alpha+[\mathrm{NII}]$ line flux corrected for atmospheric extinction. The narrowband images were obtained at the Steward Observatory Bok telescope, and the data reduction and calibration are described in Martin \& Kennicutt (1995).

${ }^{b}$ (col. 3) Logarithmic extinction at $\mathrm{H} \beta$ (Martin 1997).

${ }^{c}$ (col. 4) Intensity ratio of [NII] $\lambda 6584$ emission to $\mathrm{H} \alpha$ emission (Martin 1997).

${ }^{d}$ (col. 5) Luminosity of hydrogen ionizing photons assuming Case B recombination at $T_{e}=10^{4} \mathrm{~K}$.

${ }^{e}$ (col. 6) Formation rate of 1 to $100 \mathrm{M}_{\odot}$ stars.

${ }^{f}$ Includes only the two brightest HII regions.

${ }^{g}$ Derived from the integrated $\mathrm{H} \alpha$ luminosity (Hunter \& Plummer 1996), which is $9 \times 10^{38} \mathrm{ergs} \mathrm{s}^{-1}$ after correcting for foreground extinction. 
Table 5: Galactic Environment and Global Properties of the Feedback

\begin{tabular}{|c|c|c|c|c|c|c|c|c|c|c|}
\hline Galaxy & $\begin{array}{l}M_{d y n}{ }^{\mathrm{a}} \\
\left(\mathrm{M}_{\odot}\right)\end{array}$ & $\begin{array}{l}M_{H I} \mathrm{~b} \\
\left(\mathrm{M}_{\odot}\right)\end{array}$ & $\begin{array}{l}L_{B}{ }^{\mathrm{c}} \\
\left(\mathrm{L}_{\odot}\right)\end{array}$ & $\begin{array}{l}\text { Max Disk }{ }^{\mathrm{d}} \\
(M / L)_{*}\end{array}$ & $\begin{array}{l}L_{x} \mathrm{e} \\
(\mathrm{ergs} / \mathrm{s})\end{array}$ & $\begin{array}{l}M_{x} f^{1 / 2 \mathrm{f}} \\
\left(\mathrm{M}_{\odot}\right)\end{array}$ & $\begin{array}{l}M_{w} \sqrt{\epsilon / 0.1^{\mathrm{g}}} \\
\left(\mathrm{M}_{\odot}\right)\end{array}$ & $\begin{array}{l}d M / d t \sqrt{\epsilon / 0.1^{\mathrm{h}}} \\
\left(\mathrm{M}_{\odot} / \mathrm{yr}\right)\end{array}$ & $K E / E_{B}{ }^{\mathrm{i}}$ & $\tau_{e j} / \tau_{*} \mathrm{j}$ \\
\hline VII Zw 403 & $\ldots$ & $4.9 \mathrm{e} 7$ & $6.0 \mathrm{e} 6$ & $\cdots$ & $<2 e 38$ & $\cdots$ & $\cdots$ & 0 & $\cdots$ & $\cdots$ \\
\hline Sex A & $3.2 \mathrm{e} 8$ & $1.1 \mathrm{e} 8$ & $7.0 \mathrm{e} 6$ & 24 & $\ldots$ & $\cdots$ & $2.8 \mathrm{e} 4(0.19)$ & 0.034 & 0.0003 & 22 \\
\hline II $\mathrm{Zw} 40$ & $2.5 \mathrm{e} 9^{\mathrm{k}}$ & $4.5 \mathrm{e} 8$ & $1.3 \mathrm{e} 7$ & 144 & $\ldots$ & $\ldots$ & ... & 0 & $\ldots$ & $\ldots$ \\
\hline NGC 3738 & $\ldots$ & $1.6 \mathrm{e} 8$ & $5.1 \mathrm{e} 7$ & $\cdots$ & $\ldots$ & $\cdots$ & $2.1 \mathrm{e} 5(0.86)$ & 0.0020 & $\ldots$ & $\ldots$ \\
\hline NGC 1800 & $2.1 \mathrm{e} 9$ & $1.6 \mathrm{e} 8$ & $9.4 \mathrm{e} 7$ & 20 & $\ldots$ & $\ldots$ & $2.7 \mathrm{e} 6(0.38)$ & 0.14 & 0.006 & 2 \\
\hline NGC 2537 & $\ldots$ & $2.8 \mathrm{e} 8$ & $1.8 \mathrm{e} 8$ & $\cdots$ & $\cdots$ & $\cdots$ & $9.4 \mathrm{e} 4(0.55)$ & $\cdots$ & $\ldots$ & $\cdots$ \\
\hline NGC 3077 & $5.9 \mathrm{e} 9$ & $1.2 \mathrm{e} 9$ & $2.3 \mathrm{e} 9$ & 3.4 & $\ldots$ & $\ldots$ & $4.3 \mathrm{e} 6(0.09)$ & 0.43 & 0.001 & 6 \\
\hline NGC 4861 & 7.1e9 & $5.4 \mathrm{e} 8$ & $7.3 \mathrm{e} 7$ & 87 & $\ldots$ & $\ldots$ & $5.2 \mathrm{e} 6(0.23)$ & 0.27 & 0.004 & 1 \\
\hline NGC 5253 & $>4.3 e 8$ & $1.4 \mathrm{e} 8$ & $2.3 \mathrm{e} 8$ & $\sim 1$ & $<6.5 e 38$ & $<1.5 e 5$ & $1.2 \mathrm{e} 6(0.59)$ & 0.08 & 0.003 & $12-19$ \\
\hline NGC 4214 & $3.3 \mathrm{e} 9$ & $1.8 \mathrm{e} 9$ & $2.3 \mathrm{e} 8$ & 3.4 & $\ldots$ & $\ldots$ & $2.4 \mathrm{e} 6(0.12)$ & 0.22 & 0.0003 & 43 \\
\hline NGC 4449 & $2.6 \mathrm{e} 10$ & $2.2 \mathrm{e} 9$ & $2.8 \mathrm{e} 8$ & 80 & $6.5 \mathrm{e} 38$ & $6.1 \mathrm{e} 6$ & $9.93 \mathrm{e} 6(0.08)$ & 1.7 & 0.0008 & 2 \\
\hline
\end{tabular}

${ }^{a}(\operatorname{col} 2)$ Keplerian mass within $R_{H I}$.

${ }^{b}$ (col 3) Atomic hydrogen mass. References listed in Table 2.

${ }^{c}$ (col 4) Galactic B-band luminosity in solar units.

${ }^{d}$ (col 5) Implied stellar mass to blue light ratio in absence of dark matter, where $(M / L)_{*} \equiv\left(M_{d y n}-\right.$ $\left.1.4 M_{H I}\right) / L_{B}$ in solar units.

${ }^{e}$ (col 6) Unabsorbed soft X-ray luminosity. Point sources are excluded if they are resolved. Bandpasses and references as follows: NGC 1569: $0.5-2.0 \mathrm{keV}$ (Della Ceca et al. 1996); IZw18: $0.1-2.2 \mathrm{keV}$ (Martin 1996); M82: $0.2-3.5 \mathrm{keV}$ (Strickland et al. 1997); VIIZw403: $0.1-2.4 \mathrm{keV}$ (Papaderos et al. 1994); NGC 4449: $0.5-2.0 \mathrm{keV}$ (Della Ceca et al. 1997); and NGC 5253: $0.1-2.4 \mathrm{keV}$ (Martin \& Kennicutt 1995).

${ }^{f}(\operatorname{col} 7)$ Mass of hot, diffuse ISM defined as $M_{x}=(7 / 6) n_{e} m_{H} V$. The electron density was estimated from the spectral normalization and volume given in Della Ceca et al. 1996 (NGC 1569), Della Ceca et al. 1997 (NGC 4449), Martin \& Kennicutt 1995 (NGC 5253), and Strickland et al. 1997 (M82). All values are scaled to the distance given in Table 1 and a He:H ratio of 1:10 by number.

${ }^{g}$ (col 8) Upper limit on the total mass of warm ionized gas in large shells. i.e. The shell masses in column 8 of Table 3 have been added together assuming a filling factor $\sqrt{\epsilon / 0.1}$, but $\epsilon$ may be considerably smaller. The fractional uncertainty, in parentheses, reflects the uncertainty in the volume estimate (see $\S 4.2$ ).

${ }^{h}$ (col 9) Estimated mass ejection rate from the galactic disk, $d M / d t \equiv\left(M_{w}+M_{x}\right) / \tau_{d y n}$. Note that the contribution from hot gas is only included for M82, NGC 1569, and NGC 4449.

${ }^{i}$ (col 10) Upper limit on ratio of kinetic energy in large-scale ionized hydrogen shells to the gravitational binding energy of the atomic gas. The binding energy estimate, $E_{B} \sim\left(1.4 M_{H I}\right) v_{c}^{2}$, is a lower limit; and the kinetic energy scales as $\sqrt{\epsilon / 0.1}$ where $\epsilon \lesssim 0.1$.

${ }^{j}$ (col 11) Fraction of an orbital period to eject the entire ISM at the rate given in col. 9, where $\tau_{e j}=$ $1.4 M_{H I} / d M / d t$ and $\tau_{*}=2 \pi R\left(v_{c}\right) / v_{c}$.

${ }^{k}$ Mass of northern cloud. Southern cloud adds an additional $6 \times 10^{9} \mathrm{M}_{\odot}$. 


\section{REFERENCES}

Appleton, P. N., Davies, R. D., \& Stephenson, R. J. 1981, MNRAS, 195, 327.

Babul, A., \& Rees, M. J. 1992, MNRAS, 255, 346.

Bajaja, E., Huchtmeier, W. K., \& Klein, U. 1994, A\&A, 285, 385.

Binney, J., \& Tremaine, S. 1987, Galactic Dynamics (Princeton: Princeton Univ. Press).

Bland, J., \& Tully, B. 1988, Nature, 334, 43.

Braun, R. 1995, A\&AS, 114, 409.

Brinks, E. \& Klein, U. 1988, MNRAS, 231, 63P.

Bruzual, A. \& Charlot, S. 1993, ApJ, 405, 538.

Burbidge, E. M., Burbidge, G. R., \& Rubin, V. C. 1964, ApJ, 140, 942.

Burkert, A. 1995, ApJ, 447, L25.

Carignan, C., \& Beaulieu, S. 1989, ApJ, 347, 760.

Chiosi, C., \& Maeder, A. 1986, ARA\&R, 24, 329.

Chu, Y.-H., \& Kennicutt, R. C. 1994, ApJ, 425, 720.

Davies, R. D., Elliot, K. H., \& Meaburn, J. 1976, MNRAS, 81, 89.

de Blok, W. J. B., \& McGaugh, S. S. 1997, MNRAS, in press.

Dekel, A., \& Silk, J. 1986, ApJ, 303, 39.

Della Ceca, R., Griffiths, R. E., Heckman, T. M., \& Mackenty, J. W. 1996, ApJ, 469, 662.

Della Ceca, R., Griffiths, R. E. Heckman, T. M. 1997, ApJ, 485, 581.

ApJ, 297, 599.

de Vaucouleurs, B., de Vaucouleurs, A., Corwin, H., Buta, R., Paturel, G., \& Fouqué, P. 1991, Third Reference Catalogue of Bright Galaxies (New York: Springer-Verlag) (RC3).

de Vaucouleurs, G., de Vaucouleurs, A., \& Pence, W. 1974, ApJ, 194, L119.

DeYoung, D. S., \& Heckman, T. M. 1994, ApJ, 431, 598.

Freeman, K. C. 1970, ApJ, 160, 811.

Gallagher, J. S., Knapp, G. R., \& Hunter, D. A. 1981, AJ, 86, 344.

Cervino, M. 1997, ApJ, 483, ...

Heckman, T. M., Armus, L., \& Miley, G. K. 1990, ApJS, 74, 833 (HAM). 
Heckman, T. M., Dahlem, M., Lehnert, M. D., Fabbiano, G., Gilmore, D., \& Waller, W. H. 1995, ApJ, 448, 98.

Hill, R. S., Home, A. T., Smith, A. M., Bruhweiler, F. C., Cheng, K. P., Hintzen, P. M. N., \& Oliversen, R. J. 1994, ApJ430, 569.

Hodge, P. W. 1974, ApJ, 191, L21.

Huchtmeier, W. K., Seiradakis, J. H., Materne, J. 1981, A\&A102, 134.

Hunter, D. A. 1994, AJ, 107, 565.

Hunter, D. A., \& Gallagher, J. S. III 1990, ApJ, 362, 480.

Hunter, D., Gallagher, J., \& Rautenkranz, D. 1982, ApJS, 49, 53.

Hunter, D., A., \& Gallagher, J. S. III 1992, ApJ, 391, L9.

Hunter, D. A., \& Gallagher, J. S. III 1997, ApJ, 475, 65.

Hunter, D. A., Hawley, W. N., \& Gallagher, J. S. 1993, AJ, 106, 1797.

Hunter, D. A., van Woerden, H., \& Gallagher, J. S. 1994, AJ, 106, 1797.

Israel, F. P., \& deBruyn 1988, A\&A198, 109.

Israel, F. P., \& van Driel, W. 1990, A\&A, 236, 323.

Kennicutt, R. C. 1984, ApJ, 287, 116.

Kennicutt, R. C., Bresolin, F., Bomans, D. J., Bothun, G. D., \& Thompson, I. B. 1995, AJ, $109,594$.

Kobulnicky, H. A., \& Skillman, E. D. 1995, ApJ, 454, L121.

Kobulnicky, H. A. 1997, in preparation.

Koo, B.-C., \& McKee, C. F. 1992, ApJ, 388, 93.

Larson, R. B. 1974, MNRAS, 169, 229.

Lequeux, J., \& Viallefond, F. 1980, A\&A, 91, 269.

Leitherer, C., Vacca, W. D., Conti, P. S., Filippenko, A. V., Robert, C., Sargent, W. L. W. 1996, ApJ, 465, 717.

Mac Low, M-M, \& McCray, R., Norman, M. L. 1989, ApJ, 337, 141.

Maloney, P. \& Black, J. H. 1988, ApJ, 325, 389.

Marconi, G., Matteucci, F., \& Tosi, M. 1994, MNRAS, 270, 35.

Marlowe, A. T., Heckman, T. M., Wyse, R. F. G., \& Schommer, R. 1995, ApJ, 438, 563.

Martin, C. L., \& Kennicutt, 1995, ApJ, 447, 171. 
Martin, C. L. 1996, ApJ, 465, 680.

Martin, C. L. 1997, ApJ, Dec. 20 .

McKee, C. F. 1990, in The Evolution of the Interstellar Medium, ed. Leo Blitz, (ASP: San Francisco), p 3.

McIntyre, V. J. 1996, P.A.S.A. 14, 117.

Meaburn, J. 1979, A\&A, 75, 127.

Meaburn, J. 1980, MNRAS, 192, 365.

Meurer, G. R., Carignan, C., Beaulieu, S. R., \& Freeman, K. C., 1996, AJ, 111, 1551.

Meurer, G. R., Freeman, K. C., Dopita, M. A., \& Cacciari, C. 1992, AJ, 103, 60.

Navarro, J. F., Eke, V. R., \& Frenk, C. S. 1996, MNRAS, 283, L72.

Osterbrock, D. E. 1989, Astrophysics of Gaseous Nebulae and Active Galactic Nuclei (University Science Books: Mill Valley, CA).

of Modern Physics, 60, 1.

Papaderos, P., Fricke, K. J., Thuan, T. X., \& Loose, H.-H. 1994, A\&A, 291, L13.

Patterson, R. J., \& Thuan, T. X. 1996, ApJS, 107, 103.

Phillipps, S., \& Driver, S. 1995, MNRAS, 274, 832.

Puche, D., Westpfahl, D., \& Brinks, E. 1992, AJ, 103, 1841.

Quillen, A. C., Ramirez, S. V., \& Frogel, J. A. 1996, ApJ, 470, 790.

Reakes, M. 1980, MNRAS, 192, 297.

Reif, K., Mobold, U., Goss, W. M., vanWoerden, H., \& Siegman, B. 1982, A\&AS, 40, 451.

Rieke, G. H., 1994 Detection of Light: From the Ultraviolet to the Submillimeter (Cambridge: Cambridge University Press).

Roy, J.-R., Boulesteix, J., Joncas, G., \& Grundseth, B. 1991 ApJ, 367, 141.

Roy, J. - R., Aube, M., McCall, Marshall, L., \& Dufour, R. J. 1992, ApJ, 386, 498.

Sargent, W. L. W., \& Filippenko, A. V. 1991, AJ, 102, 107.

Shopbell, P. L., \& Bland-Hawthorn, J. 1997, astro-ph-9708038.

Shull, J. M. 1993, in Massive Stars: Their Lives in the Interstellar Medium, ed. J. P. Cassinelli \& E. B. Churchwell (San Francisco: ASP), 327.

Skillman, E. D., Terlevich, R., Teuben, P.J., \& van Woerden, H. 1988, A\&A, 198, 33.

Strickland, D. K., Ponman, T. J., \& Stevens, I. R. 1997, A\&A, 320, 378. 
Tenorio-Tagle, G., \& Bodenheimer, P. 1988, ARA\&R, 26, 145.

Thuan, T. X., \& Martin, G. E. 1981, ApJ, 247, 823.

in Starbursts and Galaxy Evolution, ed. Thuan, Montmerle, Tran Thanh Van (Editions Frontieres: Gif-sur-Yvette, France).

Toomre, A. 1963, ApJ, 138, 385.

Tully, R. B., Boesgaard, A. M., Dyck, H. M., \& Schempp, W. V. 1981, ApJ, 246, 38.

van der Hulst, J. M. 1979, A\&A, 75, 97.

van Zee, L., Westpfahl, D., Haynes, M. P., \& Salzer, J. J. 1998, AJ, ....

Vanzi, L., Rieke, G. H., Martin, C. L., \& Shields, J. C. 1996, ApJ, 466, 150.

Viallefonde, F., Lequeux, J., and Comte, G. 1987, in Starbursts and Galaxy Evolution, ed. T. X. Thuan, T. Montmerle, and J. Tran Than Van (Editions Frontière: Gif sur Yvette), p. 139.

Wang, B. 1995, ApJ, 444, 590.

Weaver, R., McCray, R., \& Castor, J. 1977, ApJ, 218, 377.

Wilcots, E. M., Lehman, C., \& Miller, B. 1996a, AJ, 111, 1575.

Wilcots, E. M., Hunter, D., Gallagher, J. S., \& Van Woerden, H. 1996b, BAAS, 188, \#61.12.

Wilcots, E. M. 1997, in prep.

Young, J. S., Allen, L., Kenney, J. D. P., Lesser, A., \& Rownd, B. 1996, AJ, 112, 1903.

Yun, M. S., Ho, P. T. P., \& Lo, K. Y. 1993, ApJ, 411, L17.

Zwicky, F. 1971, Catalogue of Selected Compact Galaxies and of Post-Eruptive Galaxies (Zurich:Speich), p. 116. 
Fig. 1.- $\mathrm{H} \alpha$ echellograms. The ticks mark increments of $46 \mathrm{~km} \mathrm{~s}^{-1}$ in the horizontal direction and $60^{\prime \prime}$ in the vertical direction. Wavelength increases to the right. The name of the galaxy and label identifying the position in Table 3 are printed at the top of each slit.

Fig. 2.- Positions of expanding shells relative to the $\mathrm{H} \alpha$ morphology. North is up, and east is to the left. Ellipses mark the locations of the Doppler ellipses identified in the echellograms. The axis of the ellipse colinear with the position angle of the slit represents the spatial dimension of the Doppler ellipse, and the second axis (usually the minor axis) is scaled such that 1 pixel is approximately $0.73 \mathrm{~km} \mathrm{~s}^{-1}$ of velocity splitting. The galaxies are: (a) NGC 3077, (b) NGC 4214, (c) NGC 4861, (d) M82, (e) NGC 3738, (f) NGC 2537, (g) NGC 2363, (h) NGC 1800, (i) Sex A, (j) NGC 5253, (k) NGC 1569, and (h) NGC 4449.

Fig. 3.- Measured radii and radial velocities of kinematic shells. For comparison, the open symbols show the kinematic shells found by Marlowe et al. (1995).

Fig. 4.- Estimated bubble ages and power requirements. The dashed lines are isochrones for adiabatic, wind-driven bubbles with radiating shells. A shell evolves parallel to a dotted line if the wind is steady. The model assumes the ambient medium is homogeneous. The curves of constant mechanical power are normalized to an ambient hydrogen number density of $n=1 \mathrm{~cm}^{-3}$, so the units for the implied power are $\left(n / 1 \mathrm{~cm}^{-3}\right) \mathrm{ergs} \mathrm{s}^{-1}$. The symbol type identifies the galaxy. The shell will be moving supersonically, $v \geq 20 \mathrm{~km} \mathrm{~s}^{-1}$, when it reaches one scale-height, $h=500 \mathrm{pc}$, if it lies above the thick dashed line (green).

Fig. 5.- Comparison of kinematic energy estimates for ionized shells. For the y-coordinate, the shell mass was derived from the bubble's volume and an ambient density $n=0.1 \mathrm{~cm}^{-3}$. For the x-coordinate, the total mass of ionized hydrogen in the complex was computed from areal $\mathrm{H} \alpha$ photometry and a filling factor for warm, ionized clouds of $\epsilon=0.1$.

Fig. 6.- Power requirements of the expanding shells versus the current mechanical power production from massive stars in the galaxy. The y-axis is scaled to an average ambient density of $0.1 \mathrm{~cm}^{-3}$. 
Fig. 7.- Rotation curve of NGC 1569. Open and starred symbols are based on the HI maps of Reakes (1980) and Israel \& van Driel (1990), respectively. The position - velocity diagrams were extracted along $\mathrm{PA}=117^{\circ}$ and deprojected assuming an inclination of $\iota=63^{\circ}$. Triangles and squares denote points to the southwest and northeast of the center of the galaxy, respectively. The dotted and dashed lines are the circular velocity curves of a Burkert halo model with core radius $r_{0}=1.4 \mathrm{kpc}$ and $532 \mathrm{pc}$, respectively. Increasing the central density by factors of 3.4 and 4.5 from Burkert's empirical $\rho_{0}\left(r_{0}\right)$ relation yields the higher circular velocities (solid line).

Fig. 8. - Escape velocity versus height above the center of the disk of NGC 1569. Solid points show the radius and projected expansion speed of the supershells for comparison. Three models for the potential are shown: (1) Dashed line - exponential disk with 500 pc scalelength and central surface density $240 \mathrm{M}_{\odot} \mathrm{pc}^{-2}$, (2) Dotted line - sum of Burkert $r_{0}=1.4 \mathrm{kpc}$ halo and exponential disk with $\alpha^{-1}=500 \mathrm{pc}, \sigma_{0}=155 \mathrm{M}_{\odot} \mathrm{pc}^{-2}$, and (3) Solid line - equal disk and halo mass within a radius of $1 \mathrm{kpc}$, same scalelengths as (2). Halo models were truncated at a radius of $160 \mathrm{kpc}$.

Fig. 9.- Rotation curves, halo escape velocity estimates, and shell expansion velocities. Open symbols show HI position - velocity data described in Table 1. Thin lines compare the rotation curves of Burkert halo models, and bold lines illustrate the halo escape velocity as a function of galactocentric radius. Only the dotted lines denote single parameter fits (Burkert's $\rho_{0}\left(r_{0}\right)$ relation). For NGC 3077 and NGC 4214, the escape velocity above a disk model is also shown, dashed lines. Solid circles are the supershells. 
This figure "fig1a.jpg" is available in "jpg" format from: http://arxiv.org/ps/astro-ph/9804165v1 
This figure "fig1b.jpg" is available in "jpg" format from: http://arxiv.org/ps/astro-ph/9804165v1 
This figure "fig1c.jpg" is available in "jpg" format from: http://arxiv.org/ps/astro-ph/9804165v1 
This figure "fig1d.jpg" is available in "jpg" format from: http://arxiv.org/ps/astro-ph/9804165v1 
This figure "figle.jpg" is available in "jpg" format from: http://arxiv.org/ps/astro-ph/9804165v1 
This figure "fig2a.jpg" is available in "jpg" format from: http://arxiv.org/ps/astro-ph/9804165v1 
This figure "fig2b.jpg" is available in "jpg" format from: http://arxiv.org/ps/astro-ph/9804165v1 
This figure "fig2c.jpg" is available in "jpg" format from: http://arxiv.org/ps/astro-ph/9804165v1 
This figure "fig2d.jpg" is available in "jpg" format from: http://arxiv.org/ps/astro-ph/9804165v1 
This figure "fig2e.jpg" is available in "jpg" format from: http://arxiv.org/ps/astro-ph/9804165v1 
This figure "fig2f.jpg" is available in "jpg" format from: http://arxiv.org/ps/astro-ph/9804165v1 
This figure "fig2g.jpg" is available in "jpg" format from: http://arxiv.org/ps/astro-ph/9804165v1 
This figure "fig2h.jpg" is available in "jpg" format from: http://arxiv.org/ps/astro-ph/9804165v1 
This figure "fig2i.jpg" is available in "jpg" format from: http://arxiv.org/ps/astro-ph/9804165v1 
This figure "fig2j.jpg" is available in "jpg" format from: http://arxiv.org/ps/astro-ph/9804165v1 
This figure "fig2l.jpg" is available in "jpg" format from: http://arxiv.org/ps/astro-ph/9804165v1 


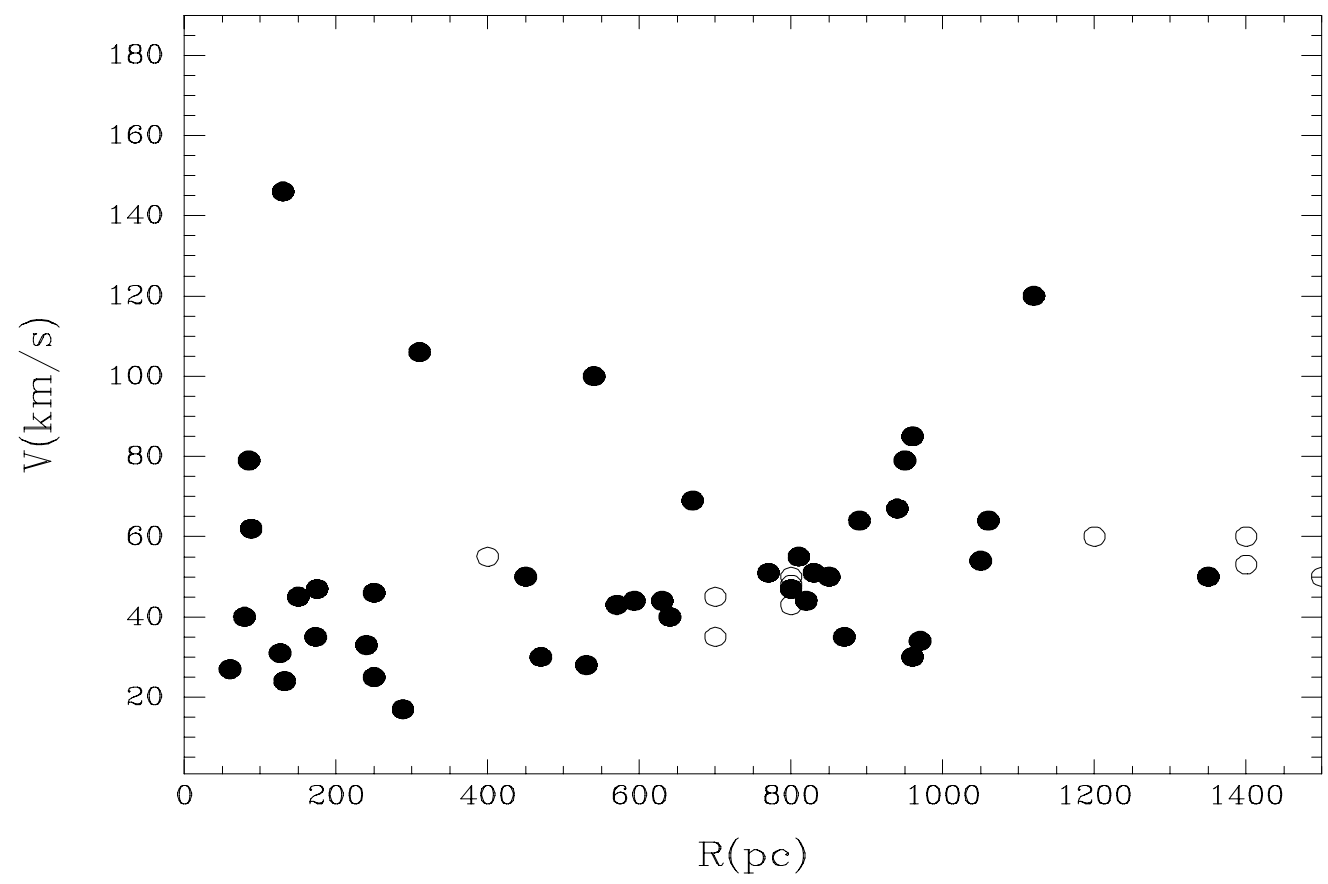




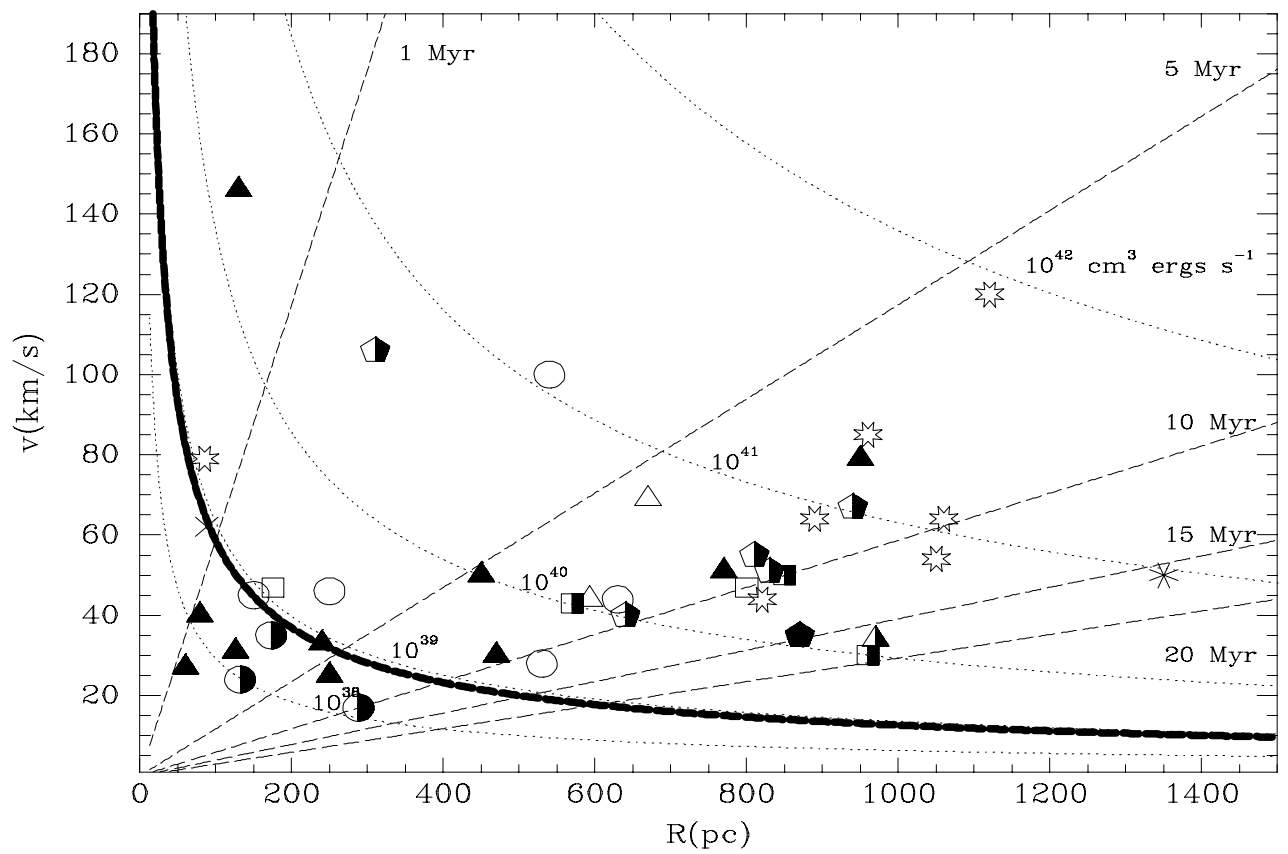

KEY:

党 NGC1569

* NGC1800

$\triangle$ NGC2363

$\square$ NGC2537

$\Delta$ I Zw 18

- NGC3077

$\times \operatorname{Sex} A$

(1) NGC3738

NGC4214

- NGC4449

- NGC4861

- NGC5253 


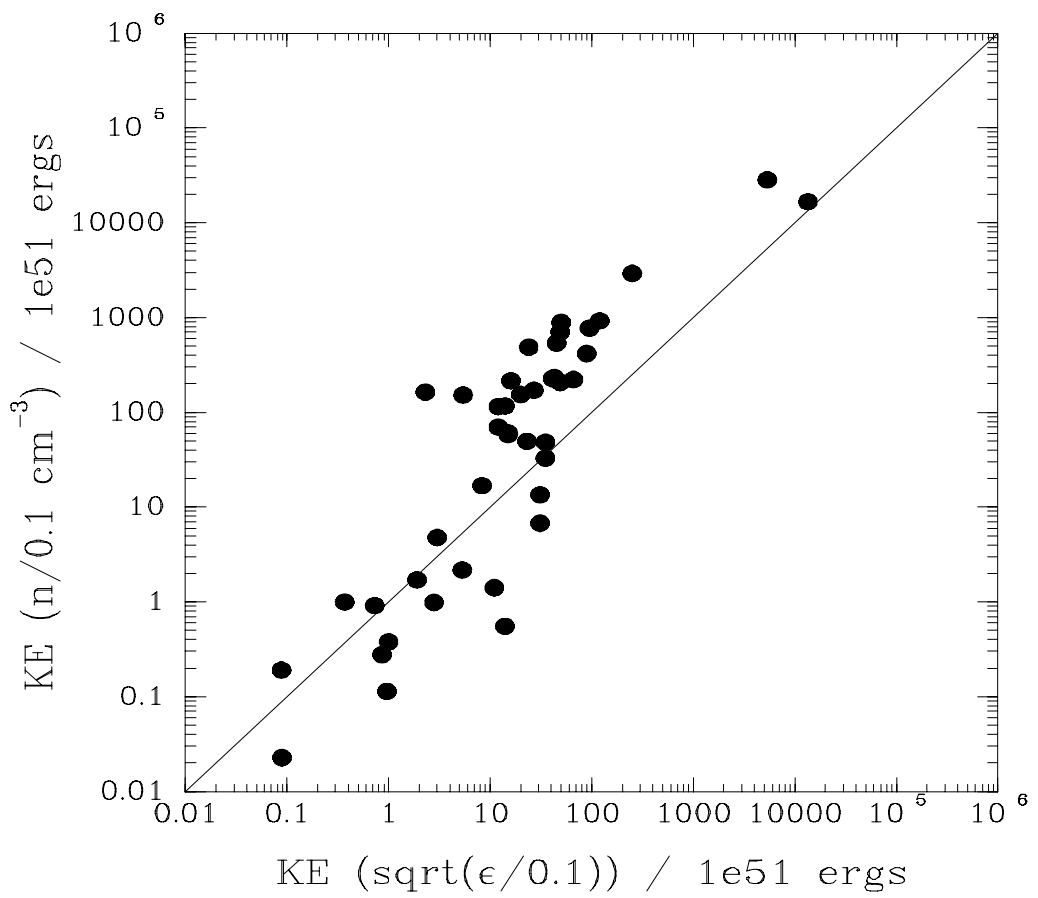




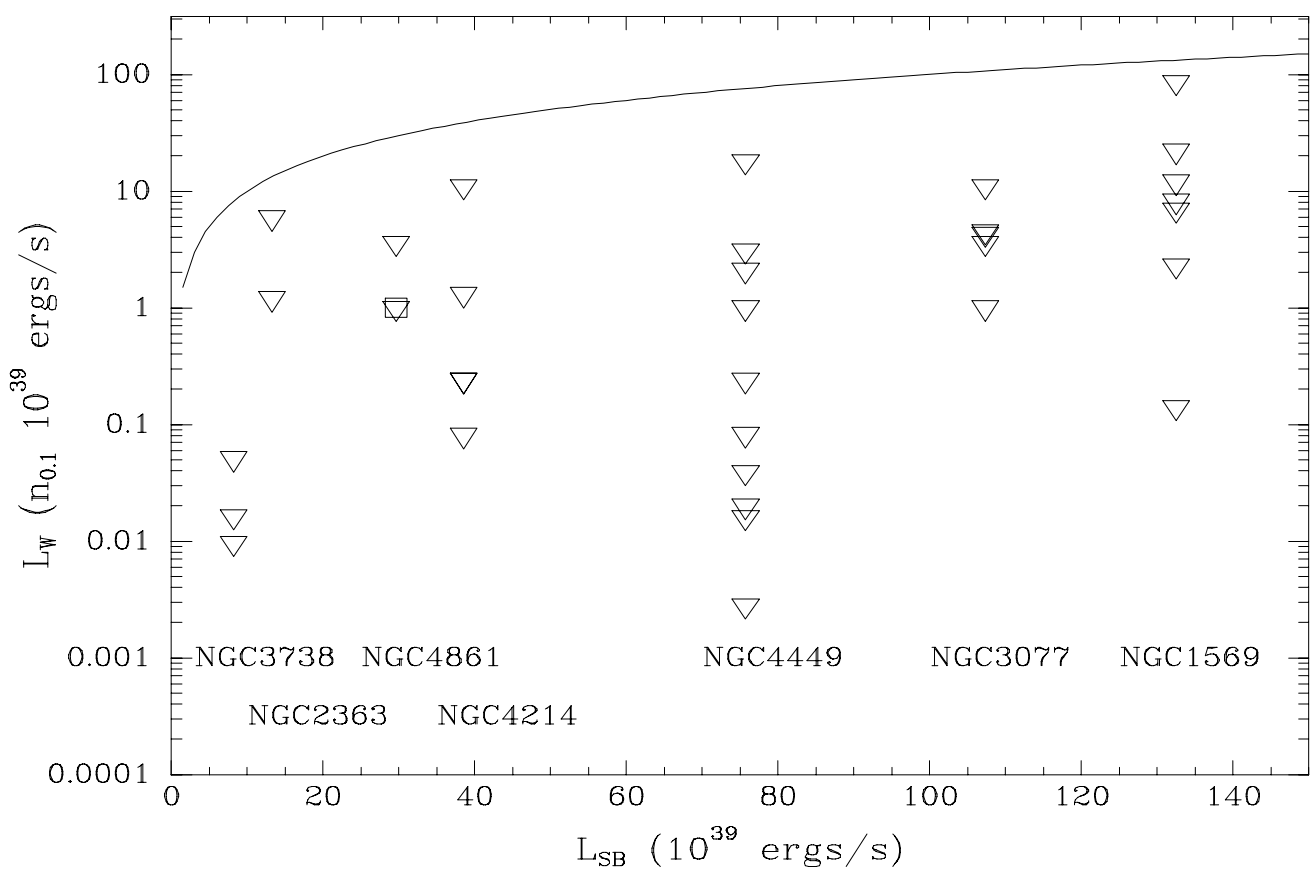




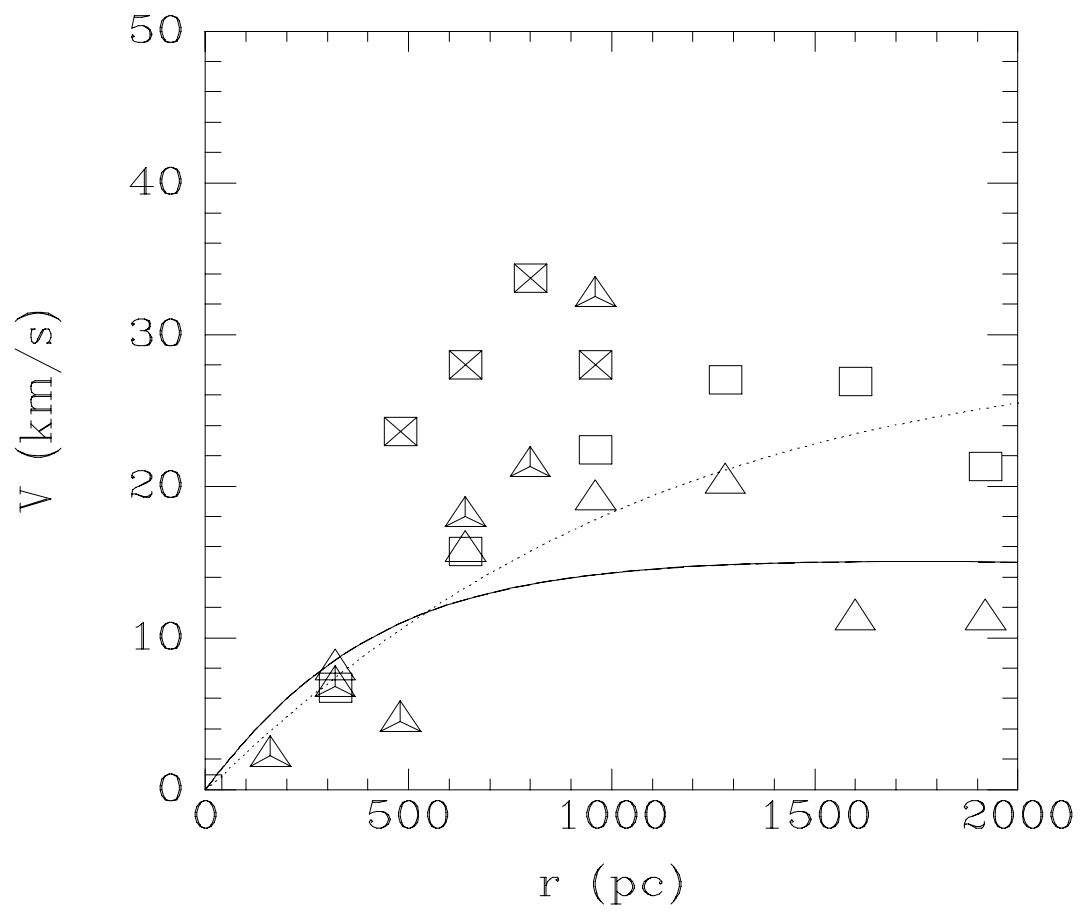




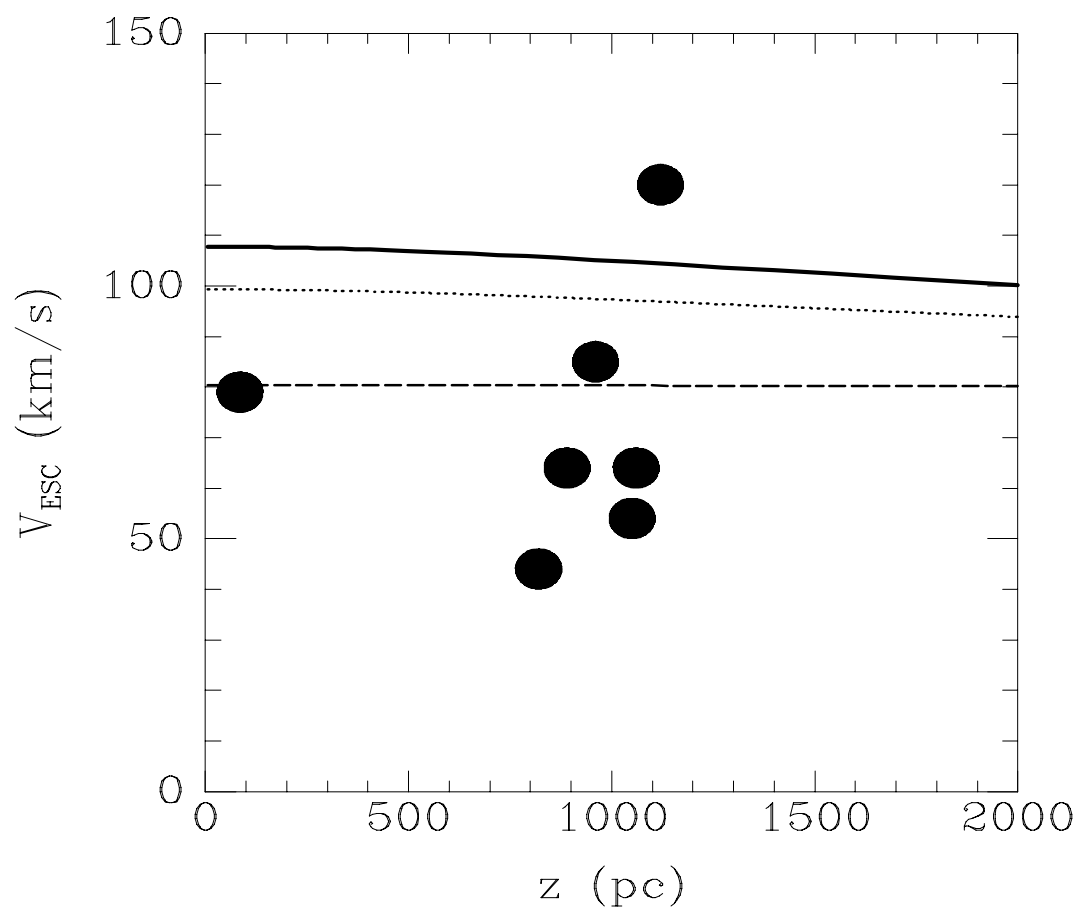



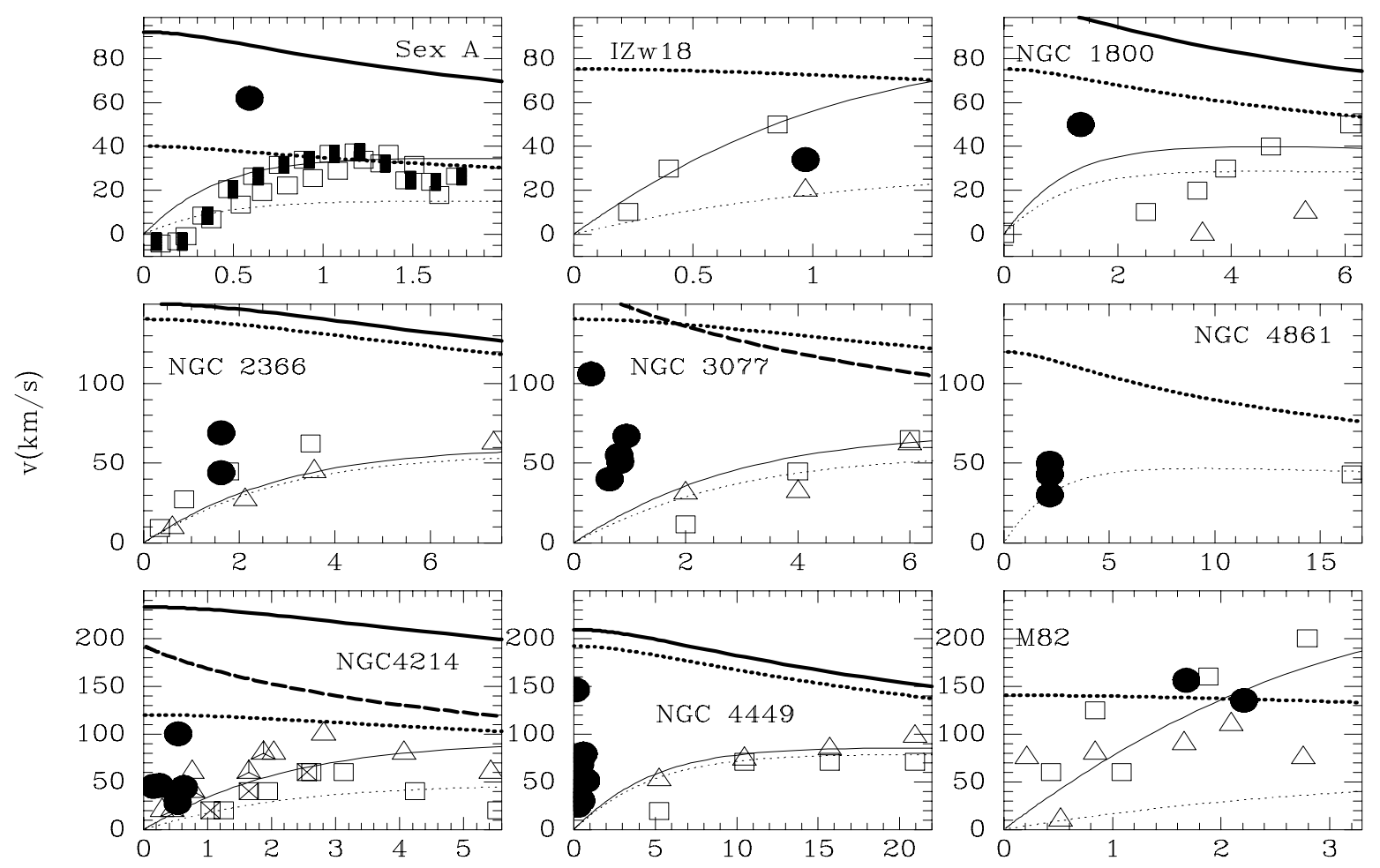

$r(k p c)$ 